\title{
A Fast-Degrading Thiol-Acrylate based Hydrogel for Cranial Regeneration
}

AM Emmakah ${ }^{1,4}$, HE Arman ${ }^{2}$, JC Bragg 3 , T Greene ${ }^{3}$, MB Alvarez 2 , PJ Childress ${ }^{2}$, WS Goebel ${ }^{4}$, MA Kacena ${ }^{2}, \mathrm{CC} \mathrm{Lin}^{1,3}$, and TM Chu ${ }^{1,2,3,5, *}$

${ }^{1}$ Weldon School of Biomedical Engineering, Purdue University, West Lafayette, IN 47908, USA

${ }^{2}$ Department of Orthopaedic Surgery, Indiana University School of Medicine, Indianapolis, IN 46202, USA

${ }^{3}$ Department of Biomedical Engineering, Indiana University - Purdue University Indianapolis, Indianapolis, IN 46202, USA

${ }^{4}$ Children's Clinical Research Center, Riley Hospital for Children, Indiana University Health, Indianapolis, IN 46202, USA

${ }^{5}$ Department of Biomedical and Applied Sciences, Indiana University School of Dentistry, Indianapolis, IN 46202, USA

* Corresponding author

Tien-Min Gabriel Chu, D.D.S., Ph.D.

Phone: 317-274-5148

Fax: 317-278-7462

Email: tgchu@iupui.edu

Short Title: PEGDA Hydrogel for Cranium Regeneration

This is the author's manuscript of the article published in final edited form as:

Emmakah, A. M., Arman, H. E., Bragg, J. C., Greene, T., Alvarez, M. B., Childress, P. J., ... Chu, T. M. (2017). A fastdegrading thiol-acrylate based hydrogel for cranial regeneration. Biomedical Materials, 12(2), 025011.

https://doi.org/10.1088/1748-605X/aa5f3e 


\begin{abstract}
Successful regeneration of the cranium in patients suffering from cranial bone defects is an integral step to restore craniofacial function. However, restoration of craniofacial structure has been challenging due to its complex geometry, limited donor site availability, and poor graft integration. To address these problems, we investigated the use of a thiol-acrylate hydrogel as a cell carrier to facilitate cranial regeneration. Thiol-acrylate hydrogels were formulated with $5-15 \mathrm{wt} \%$ poly(ethylene glycol)-diacrylate (PEGDA) and 1-9 mM dithiothreitol (DTT). The degradation rate, swelling ratio, and shear modulus of the resulting hydrogel were first characterized. Then, preosteoblast-like cells (MC3T3-E1) were encapsulated in the hydrogel and cultured for up to 21 days. Our results demonstrate that compared to samples formulated from $15 \mathrm{wt} \%$ PEGDA, $5 \mathrm{wt} \%$ PEGDA samples showed lower storage modulus at day $10(0.7 \mathrm{kPa}$ vs. $8.3 \mathrm{kPa}), 62.7 \%$ higher in weight change after soaking for 10 days. While $5 \mathrm{wt} \%$ PEGDA group showed an $85 \%$ weight loss between day 10 and 21, $15 \mathrm{wt} \%$ PEGDA group showed a 5\% weight gain in the same time period. Cell viability with $15 \mathrm{wt} \%$ PEGDA and $5 \mathrm{mM}$ DTT hydrogel decreased $41.3 \%$ compared to $5 \mathrm{wt} \%$ PEGDA and 5mM DTT gel at day 7. However, histological analysis of cells after 21 days in culture revealed that they had pericellular mineral deposition indicating that the cells were differentiating into osteoblasts lineage in all experimental groups. This study shows that thiol-acrylate hydrogels can be tailored to achieve different degradation rates, in order to enhance cell viability and differentiation. Thus, the findings of this study provide a fundamental understanding for the application of thiol-acrylate hydrogels in cranial bone regeneration.
\end{abstract}

Keywords: PEGDA hydrogel, cranium regeneration, thiol-acrylate, 3D cell culture, cell differentiation, osteoblasts

PACS Classification Number: 87.85.J- 


\section{Introduction}

Biodegradable hydrogels are an attractive class of engineered materials that have been widely used in several medical fields. These materials have gained wide attention in the tissue engineering field due to their resorbability and potential as cellular carriers and/or drug delivery vehicles (Reddy et al., 2005, Yang et al., 2010). In the tissue engineering field, there is a sustained effort to design and develop hydrogel platforms with appropriate mechanical properties and a predictable degradation behavior for multiple applications (Shih and Lin, 2012). Many classes of hydrogels have been explored for these applications and among them the poly(ethylene glycol) (PEG)- hydrogel has attracted the attention of many biomaterial engineers due to the easy access of the commercially available macromers as well as the simplicity in macromer synthesis. Additionally, many applications desire the flexibility of PEG-hydrogels that allow the addition of a variety of termini during synthesis (Hern and Hubbell, 1998, Bryant et al., 2000, Elbert and Hubbell, 2001, Ward and Peppas, 2001, Lin-Gibson et al., 2004, Weber et al., 2006, Hao et al., 2014, Lin and Anseth, 2009a, Bahney et al., 2011). Previously, visible light-mediated crosslinking of degradable hydrogels was developed by using linear poly(ethylene glycol)-diacrylate (PEGDA) as the backbone macromer, non-cleavage type photosensitizer eosin $\mathrm{Y}$ as the initiator, and bifunctional dithiothreitol (DTT) as the co-initiator and crosslinker (Hao and Lin, 2014, Hao et al., 2014). In this system, the incorporation of thiol groups into the PEGDA hydrogel resulted in a thiolether-ester bond that allows bulk hydrolytic degradation (Lin et al., 2015). Because the PEGDA backbone lacks biological motifs, the cell adhesion peptide, Cys-Arg-Gly-Asp-Ser (CRGDS), was added to the prepolymer solution for incorporation into the network structure (Hern and Hubbell, 1998, Sokic and Papavasiliou, 2012, Li and Kao, 2003, Yang et al., 2005). Furthermore, a comonomer, $N$-vinylpyrrolidone (NVP) was added to the prepolymer solution, not only to accelerate the gelation process, but also to enhance the mechanical stiffness of the hydrogel (Fratzl-Zelman et al., 1998, Sokic and Papavasiliou, 2012, Shih and Lin, 2012, Papavasiliou et al., 2008). This visible light thiol-acrylate system uses a mixed-mode (step-and-chain) growth mechanism (Hao et 
al., 2014, Hao and Lin, 2014). Under these conditions, a thiyl radical generated by a proton extracted from a photo-excited eosin Y, attacks the vinyl groups present on PEGDA to generate a carbon radical, which propagates through another acrylate to permit chain-transfer events or extracts hydrogen from another thiol to permit a step-growth thiol reaction (Hao and Lin, 2014). The advantage of this gelation mechanism over other thiol-acrylate gelation mechanisms, is the use of a biocompatible, non-cleavage, visible light initiator eosin Y. Furthermore, in this system, the bifunctional thiol, DTT, serves both as a cytocompatible co-initiator and a cross-linker of the PEGDA hydrogel matrix. The use of DTT and eosin Y to initiate and promote gelation has unique advantages compared to other initiators used to initiate gelation processes. The most important is the toxicity generated by cleavage type initiators, such as I-2959, or the co-initiators such as triethanolamine (Hao and Lin, 2014, Shih and Lin, 2012, Hao et al., 2014).

Degradation rate of a hydrogel cell carrier is critical for the success of cell delivery. The carrier should last long enough for the encapsulated cells to proliferate and differentiate; however, it should degrade in time and allow native tissue matrices to develop (Anseth et al., 2002, Bryant and Anseth, 2002, Lin and Anseth, 2009b). The advantage of using a synthetic hydrogel is the ability to design and control the degradation rate based on a target application. In previous studies, several parameters such as the concentrations of the co-monomers NVP, DTT, and the types of pendent peptide were shown to affect the degradation behaviors of hydrolytically degradable PEGDA hydrogels (Hao and Lin, 2014, Lin et al., 2015, Hao et al., 2014). It is known that the cellmaterial interaction is dependent on the type of cells encapsulated within the hydrogels. Although promising results have been reported with encapsulated hepatocytes(Hao and Lin, 2014), additional investigation is required to tailor the gel properties in order to explore the use of appropriate cell types for bone regeneration.

The aim of this study is to explore the use of photopolymerized PEGDA hydrogel for a non-load bearing bone defect application. Although great progress has been made on the use of PEGDA hydrogels, additional studies are needed to tailor and improve these hydrogels. In this 
study, we examine the effects of PEGDA macromers and the concentration of DTT on the mechanical stiffness and degradation rate of the formed hydrogel in the context of cranial tissue engineering. The cytocompatibility of thiol-acrylate hydrogel is also evaluated using in situ encapsulation and differentiation of a pre-osteoblast like cell line (MC3T3-E1).

\section{Materials and Methods}

\subsection{Materials}

PEG (6 kDa), DTT, N-vinylpyrrolidone (NVP), eosin-Y disodium salt, von Kossa staining kit, ascorbic acid, and $\beta$-glycerophosphate disodium salt hydrate were purchased from SigmaAldrich (St. Louis, MO, USA). Peptide sequence (CRGDS) was purchased from Thermo Fisher Scientific (Rockford, IL, USA). Dulbecco's Phosphate-Buffered Saline (DPBS) and 0.25\% Trypsin were purchased from Fisher Scientific (Fisher Scientific, Indianapolis, IN). AlamarBlue cell metabolic activity kit was obtained from AbD Serotec (Raleigh, NC, USA). Live/dead staining kit and Minimum Essential Medium Eagle Alpha ( $\alpha$ MEM) were obtained from Life Technologies (Grand Island, NY, USA).

\subsection{Methods}

\subsubsection{Poly(ethylene glycol)-diacrylate Macromer Synthesis}

PEGDA was synthesized following an established protocol as described previously (Hao et al., 2014, Hao and Lin, 2014). Briefly, chemical reactions were completed between azeotropically dried PEG-hydroxyl (PEG-OH) and triethylamine, by adding the later slowly into the PEG/toluene solution with continuous stirring. Following the initial reaction, acryloyl chloride was added to the PEG/toluene solution in drops through an addition funnel. The reaction was allowed overnight in the dark in the presence of nitrogen. To remove the triethylamine salt, the 
mixed solution was filtered using a flask containing neutral aluminum oxide and sodium carbonate. Then, the heterogeneous solution was stirred for $2 \mathrm{~h}$, followed by filtration via a thin layer of Hyflo, rotovap, and precipitated in cold ether and the extracted PEGDA macromers were characterized by HNMR (AVANCE Bruker 500). All PEGDA used in this study had a degree of functionalization between $93-96 \%$.

\subsubsection{Hydrogel Swelling Ratio and Degradation}

Thiol-acrylate hydrogel was prepared by visible light initiated photopolymerization. Briefly, a stock solution containing the photo-sensitizer (Eosin-Y), co-monomer (NVP), and cell attachment peptides (CRGDS) was prepared in 1X DPBS at pH 7.4. PEGDA macromers were added to the stock solution to obtain final concentrations of $5,7,9,12$, or $15 \mathrm{wt} \%$. DTT was then added to obtain solutions with final concentrations of $1,3,5$, 7, or $9 \mathrm{mM}$. In all solutions, the final concentrations of all other components remained identical ( $0.67 \mathrm{mM}$ of CRGDS peptide, $0.1 \mathrm{mM}$ of Eosin-Y, and $0.2 \%$ of total solution volume of NVP). Each hydrogel sample was prepared by adding $25 \mu \mathrm{l}$ of pre-polymer solution containing different concentrations of PEGDA macromers and DTT into a $1 \mathrm{~mL}$ topless cylinder shaped syringe. Then, the samples were exposed to halogen cold light (400-700 nm, AmScope, Inc.) for 9 min at 90 KLux. For each group (3 samples per group), hydrogels were prepared. The hydrogel samples from each group were then dried in a vacuum desiccator for $48 \mathrm{~h}$, weighed, and soaked in DPBS (pH 7.4) and maintained in a 95\% humidified air incubator at $37^{\circ} \mathrm{C}$. After $4 \mathrm{~h}$, the samples were weighed to record day 1 data. Subsequently, PEGDA samples were weighed every $24 \mathrm{~h}$ for 21 days. The weight change percentage of the soaked gel $(\mathrm{q})$ was calculated from the weights of the swollen sample $\left(\mathrm{W}_{\mathrm{t}}\right)$ and the dry sample $\left(\mathrm{W}_{\mathrm{o}}\right)$ (Eq. 1).

$\mathrm{q}=\frac{(\mathrm{Wt}-\mathrm{Wo})}{\mathrm{Wo}_{\mathrm{o}}}$ 


\subsubsection{Oscillation Rheometry}

To characterize gel stiffness, a Bohlin CVO 100 digital rheometer was used. Samples were prepared by injecting aliquots of pre-polymer solutions containing various amounts of PEGDA macromers $(5,7,9,12$ or 15 wt\%), 0.67 mM CRGDS, $0.1 \mathrm{mM}$ Eosin-Y, 0.2 volume\% NVP, and $5 \mathrm{mM}$ DTT onto glass slides separated by $1 \mathrm{~mm}$ Teflon spacers. After gelation, disc-shaped hydrogels with $8 \mathrm{~mm}$ diameter were created using a biopsy punch. The discs were then transferred into 6-well plates containing DPBS (pH. 7.4) and incubated at $37 \mathrm{C}^{\circ}$. Shear modulus (G') and loss modulus (G") of hydrogel discs from each group were measured at predetermined time-points (days, $1,3,5,8$, and 10$)$ by using an oscillatory rheometry operating in strain sweep mode $(0.1-$ $5 \%)$ at $1 \mathrm{~Hz}$ frequency, using a parallel plate geometry $(8 \mathrm{~mm})$ with a gap size of $800 \mu \mathrm{m}$. The average values of G' and G' were obtained from the linear portion of the shear modulus-strain curves (D'Este and Eglin, 2013, Zustiak and Leach, 2010).

\subsubsection{MC3T3-E1 Cell Culture and Encapsulation}

Pre-osteoblast like cells (MC3T3-E1 cells, ATCC®, Manassas, VA, USA) at passage 4 were used in this study. In brief, cells were cultured in $150 \mathrm{ml}$ basal $\alpha \mathrm{MEM}$ containing $10 \%$ Fetal Bovine Serum (FBS, HyClone ${ }^{\circledR}$ ), 1\% penicillin/streptomycin (HyClone ${ }^{\circledR}$, Logan, Utah, USA), and $1 \mu \mathrm{g} / \mathrm{mL}$ amphotericin B (Life Technologies). Cells were maintained at $37 \mathrm{C}^{\circ}$ and $5 \% \mathrm{CO}_{2}$ in an incubator with 95\% humidity. Culture medium was refreshed twice every week.

MC3T3-E1 cells were collected at $80 \%$ confluency by trypsinization and suspended in sterile 1X DPBS ( $\mathrm{pH} 7.4$ ). Then, $2 \times 10^{6}$ cells pooled from four samples per group were mixed in previously prepared (see above) pre-polymer solutions containing various amounts of PEGDA macromers $(5,7,9,12$ or $15 \mathrm{wt} \%), 0.67 \mathrm{mM}$ CRGDS, $0.1 \mathrm{mM}$ Eosin-Y, 0.2 volume\% NVP, and $5 \mathrm{mM}$ DTT. From each group, aliquots of $25 \mu \mathrm{L}$ well-mixed pre-polymer solution with cells were 
injected into $1 \mathrm{~mL}$ topless syringes. The samples were then exposed to cold halogen lamp illumination for $9 \mathrm{~min}$ at $90 \mathrm{kLux}$ to form a cylinder shaped gel that was $2 \mathrm{~mm}$ high and $4.5 \mathrm{~mm}$ in diameter. Cell-laden hydrogels were transferred into 24-well plates and cultured in basic $\alpha \mathrm{MEM}$ or osteogenic $\alpha \mathrm{MEM}$ containing $50 \mu \mathrm{g} / \mathrm{mL}$ ascorbic acid and $10 \mathrm{mM} \beta$-glycerphosphate. These hydrogels were maintained at $37 \mathrm{C}^{\circ}$ and $5 \% \mathrm{CO}_{2}$ in an air incubator with $95 \%$ humidity. Medium was refreshed every two days. Finally, samples were harvested at various predetermined timepoints according to the experiment. All chemicals and reagents were prepared fresh on the day of the experiment.

\subsubsection{Cell Viability Assay}

Viability of encapsulated MC3T3-E1 cells was assessed quantitatively by measuring cellular metabolic activities using a 10\% AlamarBlue reagent assay. Qualitative measurement of cell viability was performed by staining hydrogels with Calcein AM Live/Dead assay (Life Technologies) and observation through a confocal microscope. At least three samples were used in each of the following experiments and each experiment was conducted three times.

\subsubsection{Metabolic Activity Assay}

On days 1,3 , and 7 , the cell culture medium was removed and hydrogels were washed three times with 1X DPBS. Next, 10\% AlamarBlue solution was added to each sample and incubated for $3 \mathrm{~h}$. Finally, $150 \mu \mathrm{l}$ from each sample was placed in a 96-well plate to measure absorption at 570 and $600 \mathrm{~nm}$ using a microplate reader.

\subsubsection{Live/Dead Assay}

On days 1 and 7, cell-laden hydrogels from each group were harvested and transferred to a 24-well plate. Then, all samples were rinsed three times with 1X DPBS and incubated for 30 min 
in a mixed buffer containing $0.25 \mu \mathrm{L} / \mathrm{mL}$ calcein $\mathrm{AM}$ (green staining for live cells), and $2 \mu \mathrm{L} / \mathrm{mL}$ ethidium homodimer-III (red staining for dead cells). After $30 \mathrm{~min}$ in the staining buffer, cell-laden gels were rinsed once with $1 \mathrm{X}$ DPBS at $37 \mathrm{C}^{\circ}$ prior to imaging using a laser scanning confocal microscope (Olympus Fluoview, FV1000). Three images were taken per hydrogel in different orthogonal.

\subsubsection{Osteogenic Differentiation of Photoencapsulated MC3T3-E1 cells}

Ability of the encapsulated MC3T3-E1 cells to differentiate into osteoblasts lineage was quantified by examining alkaline phosphatase (ALP) activity (an early marker for osteoblast differentiation) and by further histological evaluation of samples by double staining with toluidine blue to stain the nucleus and Von-Kossa to stain calcium deposits. Cell-laden hydrogels were cultured in basal $\alpha$ MEM or osteogenic $\alpha \mathrm{MEM}$, and were incubated at $37^{\circ} \mathrm{C}, 5 \% \mathrm{CO}_{2}$, and $95 \%$ humidity. The culture medium was refreshed every 2 to 3 days.

\subsubsection{Alkaline Phosphatase (ALP) Activity}

On days 1,3, and 7, cell-laden hydrogels were rinsed three times with 1X DPBS and gently transferred to $2 \mathrm{~mL}$ flat-bottom microcentrifuge tubes containing $100 \mu \mathrm{L}$ radioimmunoprecipitation assay (RIPA) lysis buffer. Then, the hydrogels were homogenized to enable lysis of cells in the gel and protein solubilization. Homogenized cell suspensions were centrifuged and the supernatants from each sample was used to measure absorbance at $405 \mathrm{~nm}$ using an Epoch plate reader (BioTek, Winooski, VT) to determine ALP activity. Finally, ALP activity was normalized to total protein concentration in each sample measured at $562 \mathrm{~nm}$.

\subsubsection{Histology: von-Kossa and toluidine blue double staining}


On day 21, photoencapsulated cells were rinsed three times with 1X DPBS, fixed in 10\% neutral buffered formalin (NBF) for $24 \mathrm{~h}$ and infiltrated with Technovit ${ }^{\circledR} 8100$ (Kulzer, South Band, IN, USA) according to the manufacturers' instructions. Samples were embedded in Peel-AWay® polyethylene plastic molds and the resulting blocks were sectioned to $5 \mu \mathrm{m}$ thick sections using a tungsten carbide knife and HM355s motorized microtome (Thermo Scientific). The sections were then mounted on charged glass slides and stained using a modified von Kossa method. Briefly, slides were placed in 5\% silver nitrate solution and exposed to blue light using the Exakt 530 drying and supplemental infiltration unit (Sigma Aldrich ) for $20 \mathrm{~min}$. Slides were then rinsed with DI water and placed in 5\% sodium thiosulfate for $5 \mathrm{~min}$. All slides were then counter-stained with an aqueous solution of $0.1 \%$ Toluidine Blue $\mathrm{O}$ for $1 \mathrm{~min}$, rinsed with DI water, air dried and cleared. Then, samples were mounted on the Eukitt ${ }^{\circledR}$ mounting medium (Sigma Aldrich). Digital images were acquired using the Aperio Digital Pathology Systems (Aperio Technologies, Park Center Drive Vista, CA).

\subsubsection{Extraction of mRNA and quantitative PCR (qPCR)}

Total RNA was isolated from encapsulated cells to determine the presence of osteoblast differentiation markers. At predetermined time points (days 1, 3, and 7), PEGDA hydrogels and the $2 \mathrm{D}$ culture controls were harvested, and rinsed three times with $1 \mathrm{X}$ DPBS. Then, the samples were transferred to $2 \mathrm{~mL}$ Eppendorf tubes and homogenized in $500 \mu \mathrm{L}$ of Trizol reagent. Next, 100 $\mu \mathrm{L}$ chloroform was added to the samples, and incubated for $10 \mathrm{~min}$ at room temperature. Later, samples were transferred to Phase Lock Gel Heavy $2 \mathrm{~mL}$ tubes (Gaithersburg, MD, USA) and centrifuged at $10,000 \mathrm{rpm}$ for $15 \mathrm{~min}$ at $4 \mathrm{C}^{\circ}$. The supernatant was then carefully transferred to a new $1.5 \mathrm{~mL}$ centrifuge tube containing $250 \mu \mathrm{L}$ isopropanol, and then centrifuged at $12,000 \mathrm{rpm}$ for 10 min at $4^{\circ} \mathrm{C}$. After removing the supernatant $1 \mathrm{~mL}$ of $75 \%$ ethanol was added to each tube, followed by vortex and centrifugation for $5 \mathrm{~min}$. The resulting supernatant was carefully removed and further cleaned by using the RNeasy Mini Kit (Qiagen) according to the manufacturer's 
protocol. Finally, the RNA was reverse transcribed to cDNA using the Transcriptor First Strand cDNA Synthesis Kit (Roche Life Science). qPCR was performed using the SYBR Master Mix (Life technology) and primers for mouse Runt-related transcription factor 2 (Runx2) and collagen type I. Glyceraldehyde-3-phosphate dehydrogenase (GAPDH) was used as the endogenous control. The reactions were carried out in a 96-well plate (MicroAmpTM, Applied Biosystems, Foster City, CA) using a qPCR system (Applied Biosystems, Foster City, CA). The sequences of primers used in qPCR are listed here.

$\begin{array}{lll}\text { Primer } & \text { Forward Sequence } & \text { Reverse Sequence } \\ \text { Runx2 } & \text { 5'-CGACAGTCCCAACTTCCTGT-3' } & \text { 5'-CGGTAACCACAGTCCCATCT-3' } \\ \text { Collagen type I } & \text { 5'-ACGTCCTGGTGAAGTTGGTC-3' } & \text { 5'-CAGGGAAGCCTCTTTCTCCT-3' } \\ \text { GAPDH } & \text { 5'-CGTGGGGCTGCCCAGAACAT-3' } & \text { 5'-TCTCCAGGCGGCACGTCAGA-3' }\end{array}$

\section{Statistical Analysis}

All data are presented as mean $\pm \mathrm{SD}$. Statistical significance of the differences between groups were determined by student's $t$-test or a one-way ANOVA, followed by Tukey's post hoc analysis. Differences between groups were considered significant at $p<0.05$. All experiments were done independently for at least three times with different cell population; prepolymer solutions were prepared fresh at every individual experiment.

\section{Results}

\subsection{Hydrogel Swelling Ratio and Degradation}

To evaluate the swelling properties and degradation behavior of PEGDA hydrogels, we compared dry and wet weights of each group over a 21 day period. Figure $1 \mathrm{~A}$ illustrates the gelation kinetic process in this study. Figure 1B illustrates the structure of the formed gel with and without cells. Figure 1C illustrate macroscopic appearance of the PEGDA hydrogels constructs with (left 
hand) and without (right hand) encapsulated MC3T3-E1 cells line. Figures 1D-H show the percentages of weight change in soaked hydrogels with increasing thiol concentrations, except for the $9 \mathrm{mM}$ DTT in the $5 \mathrm{wt} \%$ PEGDA group, where the gel did not form. Furthermore, Figures 1D$\mathrm{H}$ demonstrate the impact of bifunctional thiol concentrations and PEGDA macromers, respectively on weight change as a function of soaking time. The first region in Figures 1D-G that show hydrogels cross-linked with 5-9 mM DTT is notable between 1-15 days post-gelation. During this period, the percent weight change of hydrogels in the various groups increased over time until it reached the equilibrium stage, where cross-linked macromers can no longer extend to allow for water molecules to infiltrate. The second region shows a slight decrease in weight between 15 and 21 days post-incubation, indicating the first sign of bulk hydrolytic degradation of the network structure. On the other hand, 15wt\% PEGDA hydrogel groups cross-linked with different DTT molarities, as well as all other PEGDA groups, cross-linked with 1 and $3 \mathrm{mM}$ DTT, demonstrating a more linear relationship in the percentage weight change during the 21 days in culture. Furthermore, the most notable weight loss is seen in 5wt \% PEGDA with 5 and $7 \mathrm{nM}$ DTT and also in $7 \mathrm{wt} \%$ PEGDA with $9 \mathrm{mM}$ DTT, indicating the degradation and disintegration of the hydrogel.

Shown in Table $1 \mathrm{~A}$ is the ratio of thiol:acrylate in the 5\% PEGDA group at various DTT concentrations. It is evident that with 5wt\% PEGDA, a gel was formed in DTT concentrations at or lower than $7 \mathrm{mM}$, but not at $9 \mathrm{mM}$. These results demonstrate that a thiol:acrylate ratio of 0.84 but not 1.08 would allow gel formation. Furthermore, weight loss before 21 days in culture is observed at thiol:acrylate ratio from 0.6-0.84 .

\subsection{Oscillatory Rheometry}

Figure 2A shows the effects of PEGDA macromer content on the shear modulus of the resultant gel. Increasing PEGDA macromer concentration increased the shear moduli in all hydrogel groups. Furthermore, a gradual decrease in the shear moduli of the resultant hydrogels 
was observed over time due to hydrolytic degradation.

Figure $2 \mathrm{~B}$ shows differences between the storage moduli of $G^{\prime}$ and the respective loss moduli of $G^{\prime \prime}$ by more than one order of magnitude in each group thus confirming the success of gelation (only $5 \%, 9 \%$ and $15 \%$ groups are shown).

Overall, compared to samples formulated from 15wt\% PEGDA with $5 \mathrm{mM}$ DTT, $5 \mathrm{wt} \%$ PEGDA samples with $5 \mathrm{mM}$ DTT showed lower storage modulus at day $10(0.7 \mathrm{kPa}$ vs. $8.3 \mathrm{kPa})$ $62.7 \%$ higher in weight change after soaking for 10 days. While $5 \mathrm{wt} \%$ PEGDA group showed a $85 \%$ weight loss between day 10 and 21, $15 \mathrm{wt} \%$ PEGDA group showed a 5\% weight gain in the same time period.

\subsection{Effect of PEGDA Macromer Concentrations on Degradation Rate}

We then evaluated the degradation rate of the hydrogels through the change in shear modulus with time as described previously (Hao and Lin, 2014, Hao et al., 2014). The degradation rates in all groups followed a pseudo first order degradation kinetics equation:

$\ln \left(\frac{\mathrm{G}^{\prime}}{\mathrm{G}^{\prime} 0}\right)=-\mathrm{kt}$

where $G^{\prime}{ }_{0}$ and $G$ ' represent shear modulus of hydrogels on day 1 and at all other time-points of the experiment, respectively. $k$ is the degradation rate constant and $t$ represents the degradation time.

In Figure 3, $\operatorname{Ln}\left(\frac{G^{\prime}}{G^{\prime} 0}\right)$ was plotted as a function of time (days). The results show that the exponential decay curve of all thiol-acrylate hydrogels followed a pseudo first order degradation kinetics. Similarly, Table 2 illustrates the calculated degradation rate constants $(k)$.

\subsection{Preosteoblast Cell Viability of MC3T3-E1 Cells}




\subsubsection{Metabolic Activity Assay}

The mitochondrial metabolic activity of MC3T3-E1 cells encapsulated in hydrogels was measured by the capacity to reduce the AlamarBlue reagent. Figure 4A shows no significant differences in the cell metabolic activities of all groups on day 1 post-gelation. Conversely, on days 3 and 7 post-gelation, the $5 \mathrm{wt} \%$ PEGDA group shows significantly higher metabolic activities than the other PEGDA hydrogel groups. Interestingly, the metabolic activity in the $15 \mathrm{wt} \%$ PEGDA group remained similar at all time-points, with no statistically significant difference found on days 1,3 and 7.

\subsubsection{Live/Dead Assay}

Figure 4B shows the viability of MC3T3-E1 cells encapsulated in hydrogels containing different concentrations of PEGDA macromers. High cell viability can be observed in all PEGDA hydrogel groups on day 1 with only small amount of dead cells (red cells) visible in the 12 and 15 $w t \%$ groups ( 3 samples for each group were imaged, each sample was imaged in at least 3 different areas). Furthermore, live/dead staining revealed that cells tend to form small clusters with increasing PEGDA macromer concentrations. However, higher cell viability (more green cells) was observed in the 5 and $7 \mathrm{wt} \%$ PEGDA groups on day 7 compared to the 9, 12, and $15 \mathrm{wt} \%$ PEGDA groups, which contained more dead cells (red).

\subsection{Osteogenic Differentiation of Photoencapsulated MC3T3-E1 cells}

\subsubsection{Alkaline Phosphatase (ALP) Activity}

Figure 5A shows the effect of osteogenic induction on the ALP activity of MC3T3-E1 cells encapsulated in 5\% and 15\% PEGDA gels for 7 days. Statistically significant difference is not observed in the ALP activities of MC3T3-E1 encapsulated in 5 $\mathrm{wt} \%$ and $15 \mathrm{wt} \%$ PEGDA on days 1 and 3. The positive control group with a 2D cell culture illustrates significantly higher ALP activity 
compared to the $15 \mathrm{wt} \%$ PEGDA group on day 7 . Nevertheless, there is no significant difference between the cells in $2 \mathrm{D}$ culture and those in $5 \mathrm{wt} \%$ PEGDA on day 7.

\subsubsection{Histological Sectioning: von-Kossa and toluidine blue double staining}

Figure 5B shows the histological sections of MC3T3-E1 cells encapsulated in PEGDA hydrogels. Von Kossa staining was used to investigate calcium deposits (black) and toluidine blue was used to label nuclei (blue). As expected, all groups cultured in the osteogenic medium demonstrated pericellular calcium deposits proximal to the blue stained cell nuclei. Since $5 \mathrm{wt} \%$ PEGDA is a fast degrading hydrogel, cells migrated into nearest surface (dish culture surface) to continue to differentiate and to deposit sufficient amounts of calcium during gel degradation. In contrast, no calcium staining is observed in groups cultured in basal medium, although toluidine blue stained cell nuclei are clearly visible.

\subsection{Reverse Transcription-quantitative Polymerase Chain Reaction (RT-qPCR)}

To further examine cell differentiation status, we preformed RT-qPCR to monitor the expression of two osteogenic genes, Runx2, and collagen type I, in MC3T3-E1 cells encapsulated in $5 \mathrm{wt} \%$ PEGDA, $15 \mathrm{wt} \%$ PEGDA, and in cells in the 2D culture positive control. Figure 6A shows the expression of Runx 2 at three early time-points, day 1, day 3, and day 7, in cells cultured in the osteogenic differentiation medium. Although no significant difference can be observed between the groups on days 1 and 3 post-gelation, Runx 2 expression is significantly upregulated on day 7 in MC3T3-E1 cells encapsulated in $5 \mathrm{wt} \%$ PEGDA compared to $15 \mathrm{wt} \%$ PEGDA ( $\mathrm{p}<$ $0.05)$ and the 2D culture $(\mathrm{p}<0.01)$ groups. The expression of Runx2 increased 1.9-fold compared to the 1.25- and 0.5-fold change in the cells in the $15 \mathrm{wt} \%$ PEGDA and 2D culture groups, respectively. Interestingly, Figure 6B shows that collagen type I was significantly upregulated on day 3 in both $15 \mathrm{wt} \%$ PEGDA and 2D culture groups compared to the $5 \mathrm{wt} \%$ PEGDA group. However, on day 7 these differences between the experimental groups were not statistically 
significant.

\section{Discussion}

\subsection{Mixed-mode Thiol-acrylate Reaction}

Previously, it was reported that the gelation of visible light based thiol-acrylate hydrogel occurs through a mixed-mode step-chain growth polymerization mechanism (Hao et al., 2014). The process is initiated by exposing a prepolymer solution to visible light to allow the formation of thiyl radicals through eosin-Y-mediated proton abstraction. The thiyl radicals attack vinyl groups on PEGDA macromers to form carbonyl radicals and allow their propagation through other acrylates by chain-growth homopolymerization or by reacting with other thiols to allow step-growth addition, resulting in a mixed-mode step-chain growth polymerization mechanism (Figure 1A) (Hao et al., 2014). The incorporation of thiol groups into PEGDA hydrogel imparts a thiol-etherester bond that makes bulk hydrolytic degradation possible and increases gel swelling ratio over time (Lin et al., 2015). The mechanism of PEGDA hydrogel gelation, stiffness, and degradation depend on several factors, including the concentrations of the NVP monomers and the CRGDS peptide. In this report, we evaluated the effects of tuning the PEGDA and bifunctional thiol concentrations while maintaining the concentrations of other components stable (i.e. photosensitizer eosin-Y, NVP, and CRGDS peptide) to achieve the degradation time and stiffness needed to efficiently encapsulate cells suitable for cranial regeneration.

\subsection{Effects of Bifunctional Thiol on Swelling Kinetics and Degradation of Visible Light-Cured Thiol-Acrylate Based Hydrogel}

The network structure of thiol-acrylate hydrogel initiated by visible light photopolymerization incorporates the co-monomer NVP, thiol-containing an active peptide of 
CRGDS that is chemically bound to the hydrogel backbone through the formation of thiolether-ester linkage as seen between DTT and PEGDA backbone(Hao and Lin, 2014), and bifunctional thiol, into the PEGDA network structure. To examine the effects of PEGDA and DTT concentrations on the weight change in soaked hydrogel samples, we prepared thiol-acrylate prepolymer solutions using different concentrations of PEGDA macromers $(5,7,9,12$, and 15 $\mathrm{wt} \%)$ and various concentrations of DTT $(1,3,5,7$, and $9 \mathrm{mM})$. The concentration of all other components in the matrix were fixed (photosensitizer eosin Y - $0.1 \mathrm{mM}, \mathrm{NVP}-0.2 \%$, and CRGDS peptide $-0.67 \mathrm{mM})$.

After initiation, the bifunctional thiol can participate in the gelation process by chaingrowth polymerization between thiyl group, PEGDA and NVP macromers. As bifunctional thiol concentration increases, the chain transfer events also increase and lead to termination of the reaction or formation of the PEGDA-DTT oligomers through step-growth polymerization. As a result, the degree of homopolymerization and crosslinking among PEGDA and the number of high molecular weight polyacrylate chains decrease (Reddy et al., 2005). Therefore, decreasing PEGDA macromers and increasing the bifunctional thiol can result in increased swelling (Figure 1D-1H). At $9 \mathrm{mM}$ of DTT in the $5 \mathrm{wt} \%$ group, three-dimensional (3D) network structure no longer forms. A previous study clearly reported that NVP is required as a co-monomer to participate in the gelation of the thiol-acrylate system due to the low reactivity of the thiyl radicals generated from the di-thiol molecules by the visible light-sensitized eosin-Y (Hao et al., 2014). Therefore, when DTT level is too high, NVP levels should also be increased to allow DTT to participate in gelation. Conversely, the excessive DTT may consume NVP and prevent gel formation similar to the conditions at lower DTT concentrations.

Tuning the degradation rate of the cell carrier is important for segmental cranial defect repair. Several factors must be considered when designing a hydrogel cell carrier. The carrier should be 1) biocompatible, 2) closely match the mechanical stiffness of the target tissue, and 3) 
the carrier should degrade in a time frame that approximates new tissue formation (Chan and Leong, 2008, Zhang et al., 2014, Verhulsel et al., 2014). Figures 1D-H show the impact of bifunctional thiol concentrations and PEGDA macromers, respectively on the percentage weight change as a function of degradation time. Degradation of these hydrogels is initiated once the gel is immersed in the watery microenvironments via hydrolysis. PEGDA hydrogels tend to gain more weight and degrade faster with increasing bifunctional thiol and decreasing PEGDA macromer concentrations. This may be a result of more water molecules infiltrating into the network structure leading to an increase in weight change that increase the opportunity for hydrolytic degradation. Finally, significant weight loss occurs in the network groups with lower PEGDA macromers and higher bifunctional thiol concentrations. This can be observed in 5wt\% PEGDA with 5 and $7 \mathrm{mM}$ DTT 15 days post-gelation (Figure 1D). The 15 days experimental duration in this study that determined enough weight loss in the hydrogel to accommodate bone formation was based on the typical timeframe for fracture healing i.e., 4-6 weeks. Based on these results, only $5 \mathrm{mM}$ concentration of bifunctional thiol was chosen to evaluate stiffness and degradation rate using rheometry.

\subsection{Effect of PEGDA Macromers on the Mechanical Stiffness of Thiol-acrylate Hydrogels}

Figure 2A shows the shear moduli of hydrogels formed by various PEGDA macromer concentrations. When the concentrations of all other components (e.g. RGDC peptide, bifunctional thiol, NVP macromer, and initiator eosin Y) remained constant in the prepolymer solution, the storage moduli of the PEGDA hydrogels increase with increasing PEGDA macromer concentrations. However, a gradual decrease in the shear moduli is observed over time due to hydrolytic degradation. Previous studies have shown that the shear moduli of hydrogels are affected by the percentage of NVP macromers (Hao et al., 2014, Hao and Lin, 2014, Lin et al., 2015, Lee et al., 2014). Based on these studies, we chose $0.2 \%$ NVP peptide, which was shown to increase the viability and proliferation of hepatic cells over time (Hao and Lin, 2014). The present study illustrates the effects of PEGDA macromer concentrations on the storage moduli of hydrogels. We 
also examined how the storage and loss moduli were related in the resultant gels on day 1 postgelation (Figure 2B). The results show a one order of magnitude difference between the storage and loss moduli thus confirming the successful gelation in all 5 groups of PEGDA hydrogels.

As expected, increasing PEGDA concentration in prepolymer solutions increased the mechanical stiffness of the gels (Figure 2A). This is likely because the mesh size of the gel decreased with increasing PEGDA macromers post-gelation. The relationship between mesh size and hydrogel stiffness has been well-established, where increasing mesh size decreases mechanical stiffness and vice versa. In this system, $5 \mathrm{wt} \%$ PEGDA shows higher swelling kinetic due to increase in mesh size where more water molecules can infiltrate. However, the increasing mesh size in $5 \mathrm{wt} \%$ PEGDA hydrogels also decreased the mechanical stiffness. The opposite was observed in $15 \mathrm{wt} \%$ PEGDA where the weight change was minimal but higher mechanical stiffness is evident. Importantly, the shear modulus of 5\% PEGDA hydrogels with $5 \mathrm{mM}$ DTT at 1,886 Pa on day 1 demonstrated sufficient mechanical integrity for clinical handling.

\subsection{Effect of PEGDA Macromers on Hydrolytic Degradation of Visible Light-Cured Thiol-}

\section{Acrylate Hydrogels}

We also explored the hydrolytic kinetics of PEGDA hydrogels following thiol-acrylate photopolymerization. In this system, thiol-acrylate hydrogels degrade hydrolytically following a bulk degradation initiated by the presence of labile thiol-ether-ester bonds present throughout the swollen hydrogel network. To model the degradation behavior of hydrogels, we used a pseudo first order degradation kinetics equation (Eq. 2), which was used previously to model the degradation rates of thiol-acrylate hydrogel system (Fairbanks et al., 2009, Rydholm et al., 2005, Metters and Hubbell, 2005, Rydholm et al., 2007). These studies reported that increasing thiol concentrations in the thiol-acrylate system could increase the degradation rate of hydrogel although significant differences were not observed between all compared groups (Hao and Lin, 2014, Lin et al., 2015). 
In this study, we show that altering PEGDA macromer concentrations in thiol-acrylate hydrogels can significantly affect the degradation rate, as observed between the $5 \mathrm{wt} \%$ hydrogel and the other groups. Moreover, $5 \mathrm{wt} \%$ PEGDA hydrogels with $5 \mathrm{mM}$ DTT rapidly dissolved after day 1 postgelation. The main reason for this could be that the mesh size was larger in lower PEGDA hydrogels allowing for more water molecules to infiltrate and degrade the gel. Also, the hydrogels with lower PEGDA concentrations (5 wt \%) simply have higher $[\mathrm{SH}] /$ vinyl ratio to allow degradation.

\subsection{In Situ Encapsulation and Proliferation of MC3T3-E1 Cells in Visible Light-Cured Thiol- Acrylate Hydrogels}

A previous study on visible light-cured PEGDA hydrogels revealed their use to encapsulate Huh7 cells (a hepatocyte cell line), which maintained viability, and showed higher proliferation rate over time (Hao and Lin, 2014). The focus of this previous study was on adjusting hydrogel stiffness based on the concentration of NVP macromers and CRGDS peptides to achieve higher hepatic cell viability (Lin et al., 2015, Hao and Lin, 2014). In this study, we used MC3T3-E1 cells to demonstrate that it is feasible to maintain viability and induce differentiation in cells encapsulated in thiol-acrylate hydrogels.

The mitochondrial metabolic activity of photoencapsulated MC3T3-E1 cells in $5 \mathrm{wt} \%$ PEGDA is higher than other PEGDA hydrogel groups at all time-points. Observation through confocal microscopy revealed better cell viability in 5 and $7 \mathrm{wt} \%$ PEGDA hydrogels after seven days of culture in growth medium; fewer dead cells are seen compared to day 1 . However, in 12 and $15 \mathrm{wt} \%$ PEGDA hydrogels a notable decrease in cell viability is observed after culture for seven days. It is plausible that increasing PEGDA macromers may have reduced the mesh size, which could hinder the diffusion of nutrients into the cells (Bryant et al., 2000, Burdick et al., 2005). On the other hand, cells encapsulated in lower concentrations of PEGDA macromers allow for better exchange of oxygen and better access to growth factors due to a larger mesh size in the 
hydrogel. Another important factor related to lower cell viability in hydrogels with higher PEGDA concentration is related to free radicals. Mazzoccoli el at studied the effects of low and high PEGDA concentrations on cell viability and showed that the concentrations of free radicals resulting from the crosslinking process of PEGDA could be a main factor that decreases cell viability although other possibilities such as topology and morphology of PEGDA hydrogels cannot be ruled out (Mazzoccoli et al., 2010).

\subsection{Photoencapsulated MC3T3-E1 Cell Differentiation and Mineralization}

Designing hydrogel scaffolds in which encapsulated cells are encouraged to differentiate into osteogenic lineages is essential in bone tissue engineering. Optimal hydrogel matrices would allow for encapsulated cells to differentiate into target osteogenic lineages, and allow for ECM deposition concomitant with hydrogel degradation during an appropriate time-scale. Various methods have been studied to enhance cell differentiation in 2D and 3D cell cultures, and include designing hydrogel scaffolds with suitable mechanical strength, degradation kinetics, and biochemical cues (Wang et al., 2000, Panetta et al., 2009). In this study, we used two in vitro assays, ALP activity and mineral deposition through alizarin red staining, to evaluate osteogenic differentiation.

ALP activity in cells is an early marker for osteoblast differentiation. In the osteogenic differentiation medium, ALP activity increases in cells that are capable of differentiating down the osteoblast lineage in response to chemical cues such as ascorbic acid and $\beta$-glycerol phosphate. This has been well-established in 2D cell culture conditions. For this reason, we compared the 2D cell culture (positive control), with encapsulated MC3T3-E1 cells in soft (5 Wt\% PEGDA) and stiff (15 Wt\% PEGDA) hydrogels. Figure 5A shows a significant increase in ALP activity in $5 \mathrm{wt} \%$ PEGDA hydrogels and 2D cell culture compared to cells photoencapsulated in $15 \mathrm{wt} \%$ PEGDA hydrogels. This increase in ALP activity may be in part, due to higher cell viability in both $5 \mathrm{wt} \%$ 
PEGDA and 2D culture. Furthermore, the higher diffusion of chemical cues through the $5 \mathrm{wt} \%$ network structure compared to $15 \mathrm{wt} \%$ PEGDA could have also likely enhanced differentiation of MC3T3-E1 cells into osteoblasts lineage.

Typically, calcium deposition represents osteoblast differentiation of cells. In Figure 5B, we demonstrate the double staining of mineral deposits in photoencapsulated MC3T3-E1 in PEGDA hydrogel sections with von Kossa staining and cell nuclei with toluidine blue staining. PEGDA groups cultured in osteogenic medium show pericellular mineral deposits around the blue stained cell nuclei, indicating successful differentiation of MC3T3-E1 cells into the target osteoblast lineage. However, no calcium staining was visible in the negative control group cultured in basal medium. Yang et. al. have also shown similar induction of bone marrow stromal cells encapsulated in PEGDA hydrogel to osteoblasts lineage (Yang et al., 2005).

\subsection{Reverse Transcription-quantitative Polymerase Chain Reaction (RT-qPCR)}

Runx2 is an essential gene for osteoblast differentiation. It is upregulated during the early stage of osteoblast differentiation and its expression wanes with increased cell differentiation. Although proliferation of cells in $2 \mathrm{D}$ culture is faster compared to cells encapsulated in $5 \mathrm{wt} \%$ PEGDA (data not show), our results show that Runx2 expression is significantly upregulated in 5 wt $\%$ PEGDA compared to 2D culture on day 7 (Figure 6A). This higher expression of Runx2 in 5 $\mathrm{wt} \%$ PEGDA hydrogels shows the potential of our thiol-acrylate hydrogel as a cell carrier for cranial repair.

\section{Conclusion}

Optimizing hydrogel scaffolds that promote cell proliferation, differentiation and matrix formation is critical for tissue engineered regeneration. In this study, we have prepared and examined visible light-cured thiol-acrylate hydrogel with variable mechanical stiffness and 
degradation rates by altering PEGDA macromer concentrations in prepolymer solutions. The fast degrading thiol-acrylate hydrogel based on 5wt\% PEGDA not only supports osteoblasts lineage differentiation, but also closely matches the mechanical stiffness and degradation profile for our target application goal for non-load bearing cranial defects. Based on these findings, our future studies will further investigate its use in critical sized cranial defects regeneration in rabbit animal models.

\section{Acknowledgments}

This project was funded by the Oral and Maxillofacial Surgery Foundation (TMC) and the Osteo Science Foundation (TMC).

\section{Figure Legends}

Figure 1: (1A) Mixed-mode (Step-Chain) growth mechanism of visible light initiated thiol-acrylate photopolymerization. (1B) Illustration of formed gel structure. (1C) Illustrate macroscopic appearance of the PEGDA hydrogels constructs with (left hand) and without (right hand) encapsulated MC3T3-E1 cells line. Effects of PEGDA macromers and bifunctional thiol content on the weight change of hydrogels soaked in (1D)-5 wt\% PEGDA, (1E)-7 wt \% PEGDA, (1F)-9 $\mathrm{wt} \%$ PEGDA, (1G)-12 wt \% PEGDA, or $(1 \mathrm{H})-15 \mathrm{wt} \%$ PEGDA). $(\mathrm{N}=3$, mean $\pm \mathrm{SD})$.

Figure 2: (2A) Effects of PEGDA macromer concentrations on equilibrium shear moduli. Increasing PEGDA concentration increases share moduli at all time-points, a gradual decrease is observed in all PEGDA hydrogels due to degradation over time. (2B) A difference by one order of magnitude between the elastic modulus (G') and loss modulus ( $\left.G^{\prime}\right)$ indicates successful gelation of the hydrogel.

Figure 3: Plot of $\operatorname{Ln}\left(\mathrm{G}^{\prime} / \mathrm{G}^{\prime} 0\right)$ as a function of time. A linear relationship indicates that the degradation of thiol-acrylate hydrogels follow a pseudo first order degradation kinetics.

Figure 4: Three-dimensional culture of MC3T3-E1 cells in visible light cured PEGDA thiolacrylate hydrogels with different PEGDA macromer concentrations. (A) Cell metabolic activity on days 1, 3, and 7 for all PEGDA groups. (B) Confocal microscopic images of encapsulated MC3T3E1 cells stained with Live/Dead staining on days 1 and 7 . Scale bar is $100 \mu \mathrm{m}$. $* \mathrm{p}<0.05, * * \mathrm{p}<$ 0.01 .

Figure 5: Osteogenic differentiation of MC3T3-E1 encapsulated in PEGDA hydrogels. (A) Normalized ALP activity of encapsulated MC3T3-E1 in osteogenic medium (OST medium) and basal medium (BSL medium). (B) von Kossa staining. Cell nuclei are stained blue and calcium is stained black. Double staining demonstrates pericellular mineralization of cells cultured in 
osteogenic medium compared to PEGDA groups cultured in basal medium. Scale bar is $300 \mu \mathrm{m}$. * $\mathrm{p}<0.05$.

Figure 6: Reverse Transcription-qPolymerase Chain Reaction of MC3T3-E1cells encapsulated in PEGDA hydrogels. (A) The early osteogenesis marker, Runx2, was upregulated in the $5 \mathrm{wt} \%$ PEGDA group and the 2D MC3T3-E1 culture. (B) Collagen type I expression on day 3. ${ }^{*} \mathrm{p}<0.05$, $* * \mathrm{p}<0.01$. 
Tables:

Table 1. Formulations of Hydrogels at 5\% PEGDA

\begin{tabular}{ccccccc}
\hline $\begin{array}{c}\text { PEGDA } \\
6000 \mathrm{Da} \\
(\mathrm{wt} \%)\end{array}$ & $\begin{array}{c}\text { Acrylate } \\
(\mathrm{mM})\end{array}$ & $\begin{array}{c}\text { DTT } \\
(\mathrm{mM})\end{array}$ & {$[\mathrm{SH}] /$ Acrylate } & {$[\mathrm{SH}] /$ vinyl* $^{*}$} & Gelation & $\begin{array}{c}\text { Wt. loss } \\
\text { before } \\
600 \mathrm{hr}\end{array}$ \\
\hline 5 & 16.7 & 1 & 0.12 & 0.06 & $\mathrm{Y}$ & $\mathrm{N}$ \\
5 & 16.7 & 3 & 0.36 & 0.17 & $\mathrm{Y}$ & $\mathrm{N}$ \\
5 & 16.7 & 5 & 0.60 & 0.29 & $\mathrm{Y}$ & $\mathrm{Y}$ \\
5 & 16.7 & 7 & 0.84 & 0.40 & $\mathrm{Y}$ & $\mathrm{Y}$ \\
5 & 16.7 & 9 & 1.08 & 0.52 & $\mathrm{~N}$ & -- \\
\hline
\end{tabular}

*The vinyl group includes $\mathrm{C}=\mathrm{C}$ contribution from NVP

Table 2. Formulations of Photoencapsulated PEGDA Hydrogels incorporating bifunctional thiol with $5 \mathrm{mM}$ DTT

\begin{tabular}{cccccc}
\hline $\begin{array}{c}\text { PEGDA } \\
\begin{array}{c}6000 \mathrm{D} . \mathrm{a} \\
(\mathrm{wt} \%)\end{array}\end{array}$ & $\begin{array}{c}\text { Acrylate } \\
(\mathrm{mM})\end{array}$ & {$[\mathrm{SH}] /$ Acrylate } & {$[\mathrm{SH}] /$ vinyl* $^{*}$} & NVP/Acrylate & $\begin{array}{c}\text { Modulus } \\
\text { on Day } \\
1(\mathrm{~Pa})\end{array}$ \\
\hline 5 & 16.7 & 0.598 & 0.288 & 1.077 & 1886.70 \\
7 & 23.3 & 0.429 & 0.242 & 0.772 & 2361.13 \\
9 & 30.0 & 0.333 & 0.208 & 0.599 & 5499.81 \\
12 & 40.0 & 0.250 & 0.172 & 0.449 & 9598.19 \\
15 & 50.0 & 0.200 & 0.147 & 0.359 & 10709.29 \\
\hline
\end{tabular}

*The vinyl group includes contribution from NVP 
Table 3. Hydrolytic rate constants of the thiol-acrylate hydrogel calculated using a pseudo-first order hydrolytic degradation model

\begin{tabular}{lccccc}
\hline & 5 Wt\% & 7 Wt\% & 9 Wt\% & 12 Wt\% & 15 Wt\% \\
& PEGDA & PEGDA & PEGDA & PEGDA & PEGDA \\
\left.\hline${\text { K }\left(\text { day }^{-1}\right)}^{1}\right)$ & 0.097 & 0.010 & 0.017 & 0.023 & 0.026 \\
\hline
\end{tabular}

$1 \mathbf{A}$

\section{Initiation}

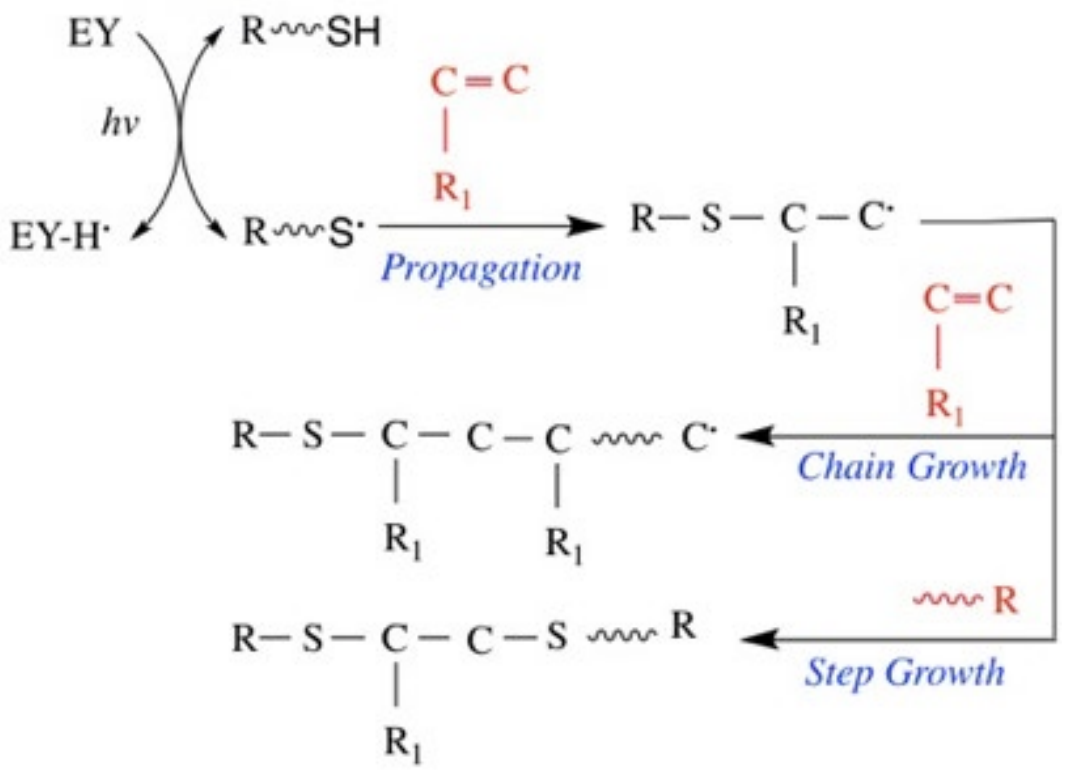

\section{B}
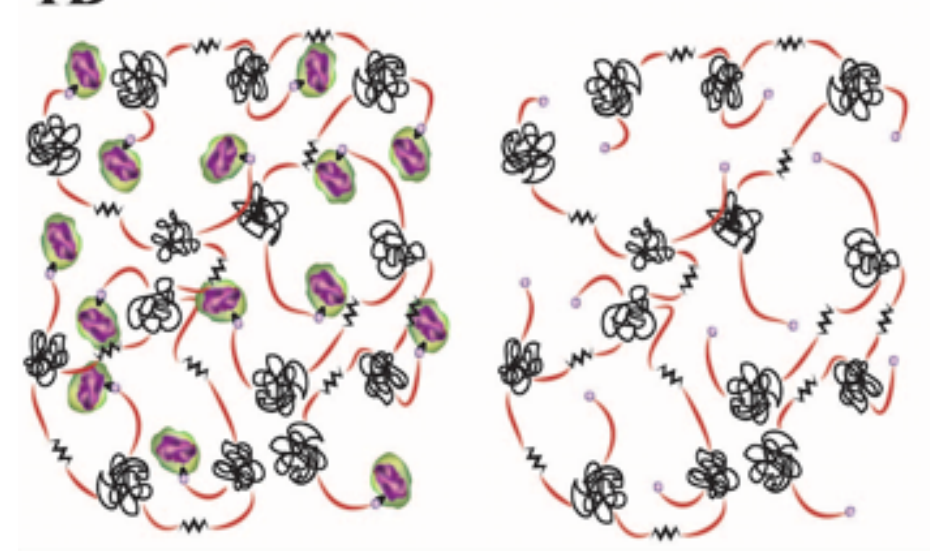

\begin{tabular}{|l|}
\hline P PEGDA \\
\hline$M$ Di-thiol linkage \\
\hline Polyacrylate \\
\hline CRGDS \\
\hline
\end{tabular}



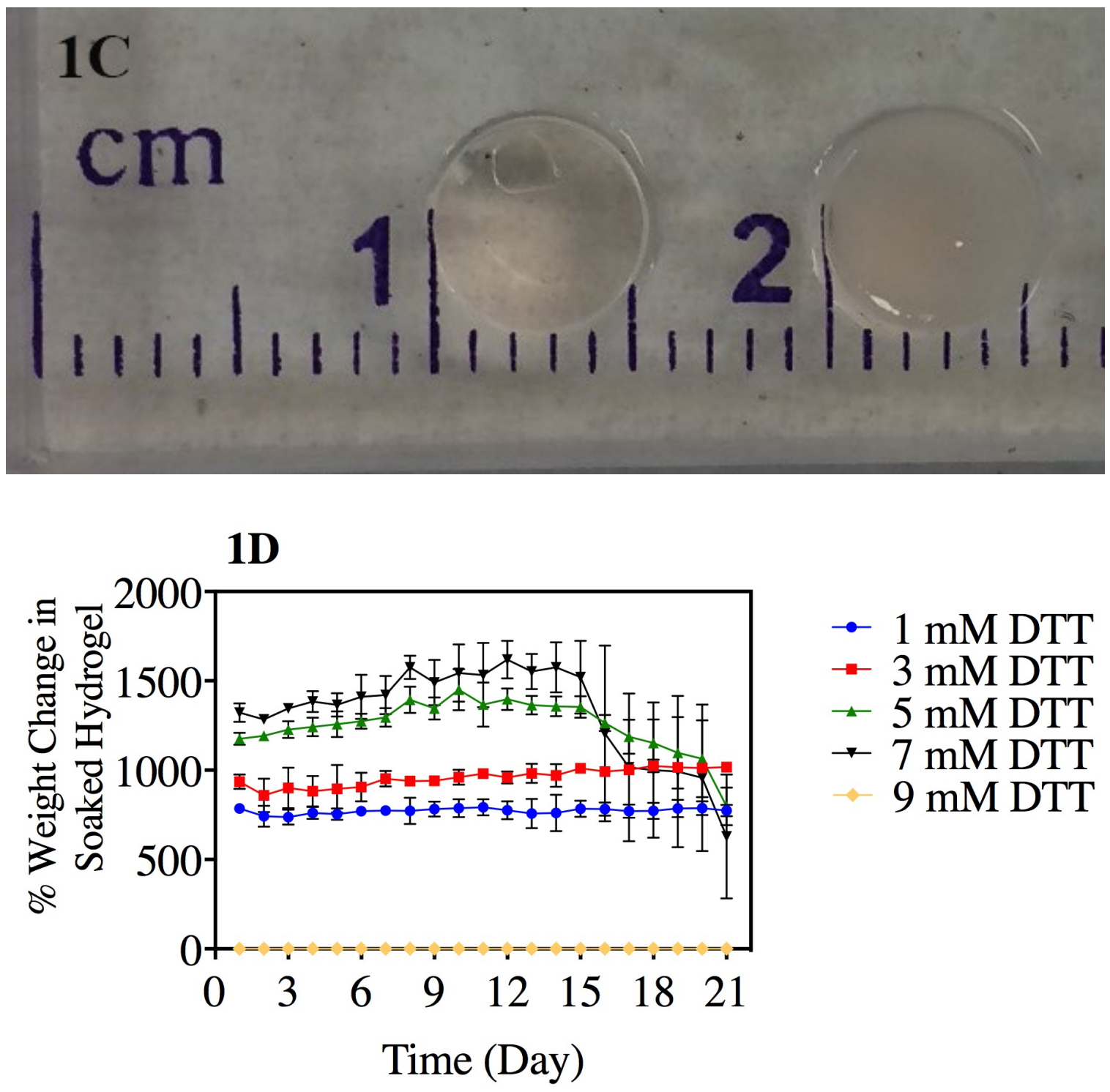

-- $1 \mathrm{mM}$ DTT

$-3 \mathrm{mM}$ DTT

$\leftarrow 5 \mathrm{mM}$ DTT

$\rightarrow 7$ mM DTT 9 mM DTT 

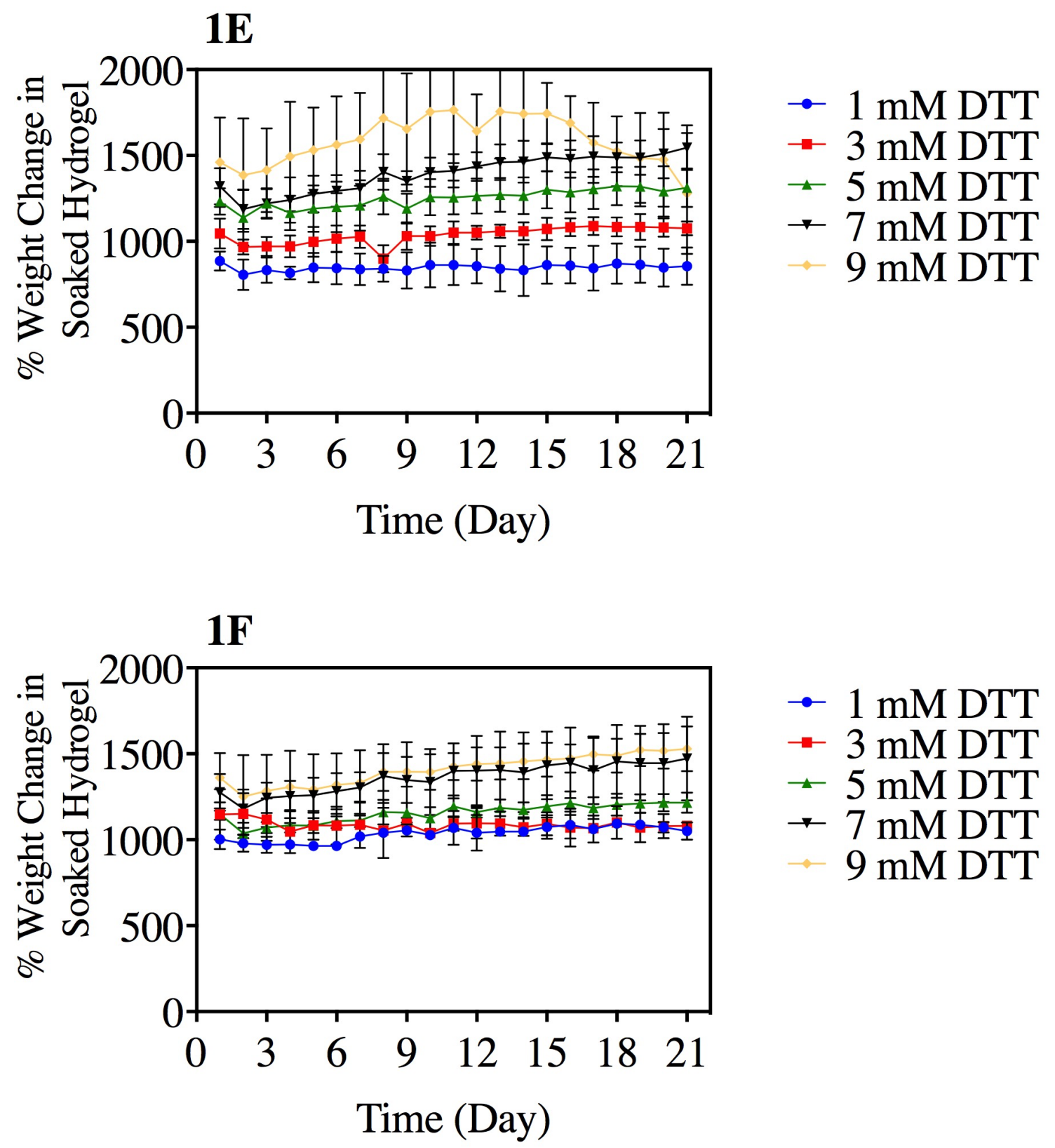

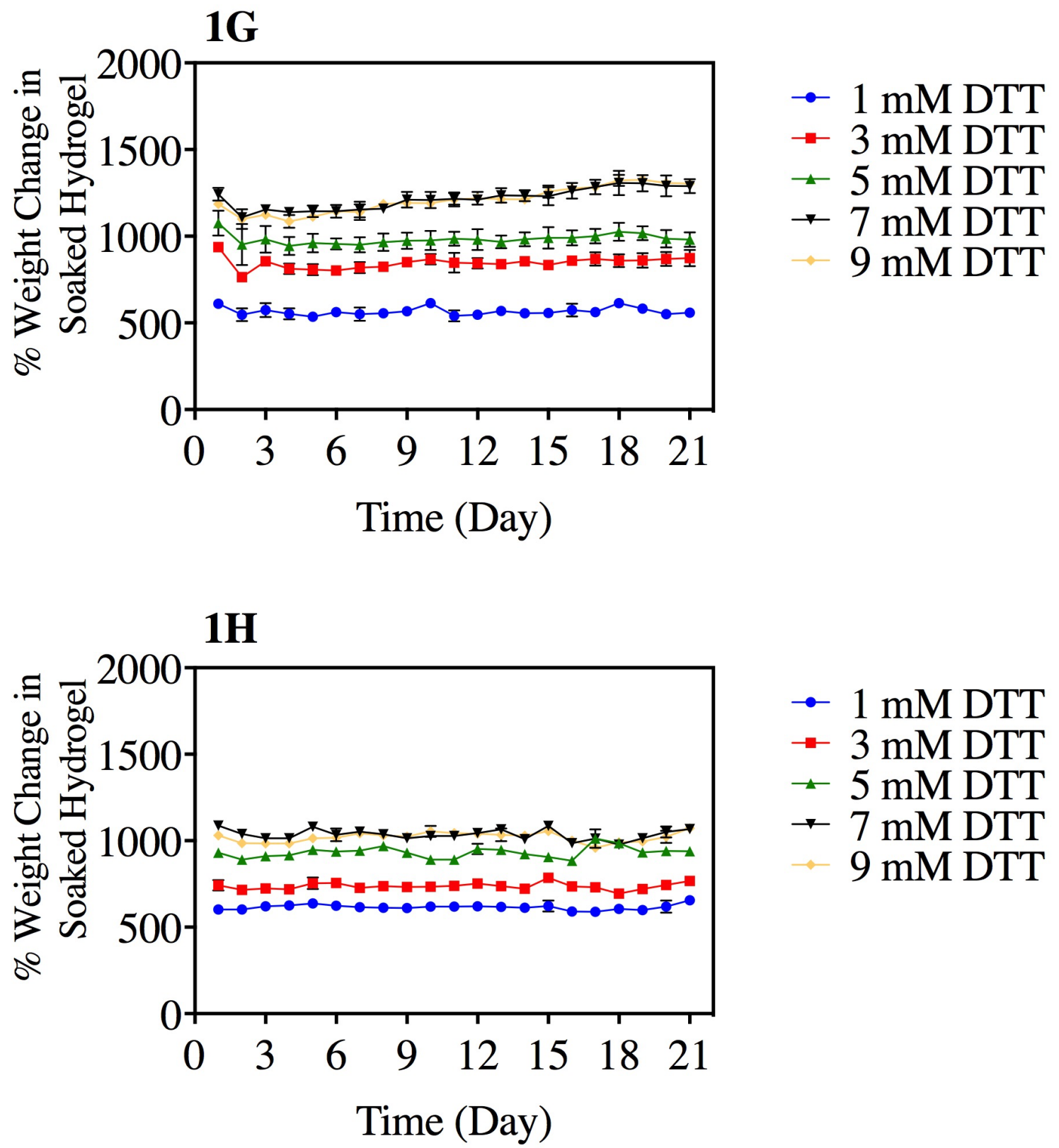

Figure 1: (1A) Mixed-mode (Step-Chain) Growth mechanism of visible light initiated thiol-acrylate photopolymerization. (1B) Illustration of formed gel structure. (1C) Illustrate macroscopic appearance of the PEGDA hydrogels constructs with (right hand) and without (left hand) encapsulated cells line (1D)-5 wt\% PEGDA, (1E)-7 wt\% PEGDA, (1F)-9 wt\% PEGDA, (1G)-12 wt\% PEGDA, (1H)-15 wt\% PEGDA) Effects of PEGDA Macromers and bifunctional thiol content on weight change in soaked hydrogel samples $(\mathrm{N}=3$, mean $\pm \mathrm{SD})$. 


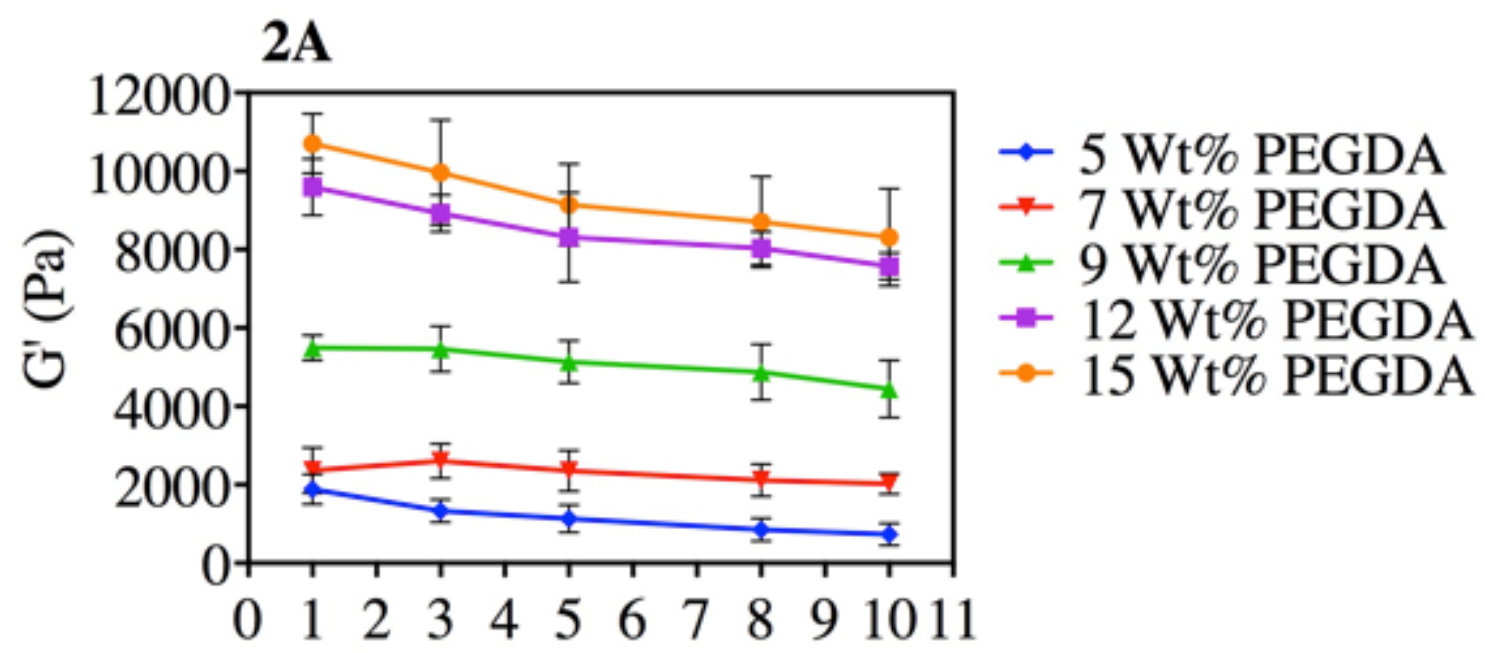

Time (Day)

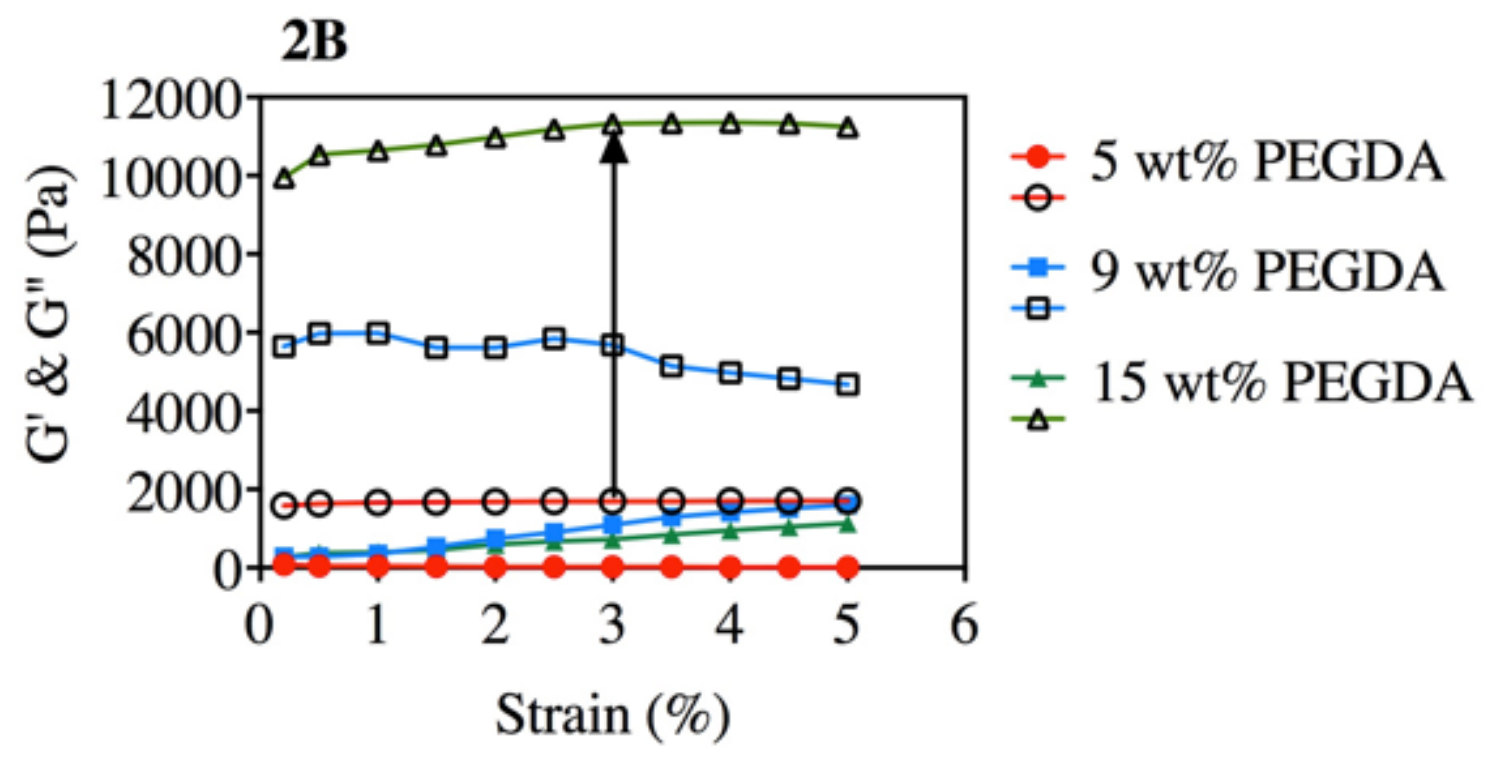

Figure 2: (2A) Effects of PEGDA Macromer Concentrations on equilibrium shear moduli. Increasing PEGDA concentration results in increasing shear moduli at all time-points, a gradual decrease is seen in all PEGDA hydrogel groups due to degradation over time. (2B) A difference of one order of magnitude between elastic modulus ( $G^{\prime}$, shown in filled symbols) and loss modulus (G", shown in empty symbols) indicates successful gelation in the hydrogel. 


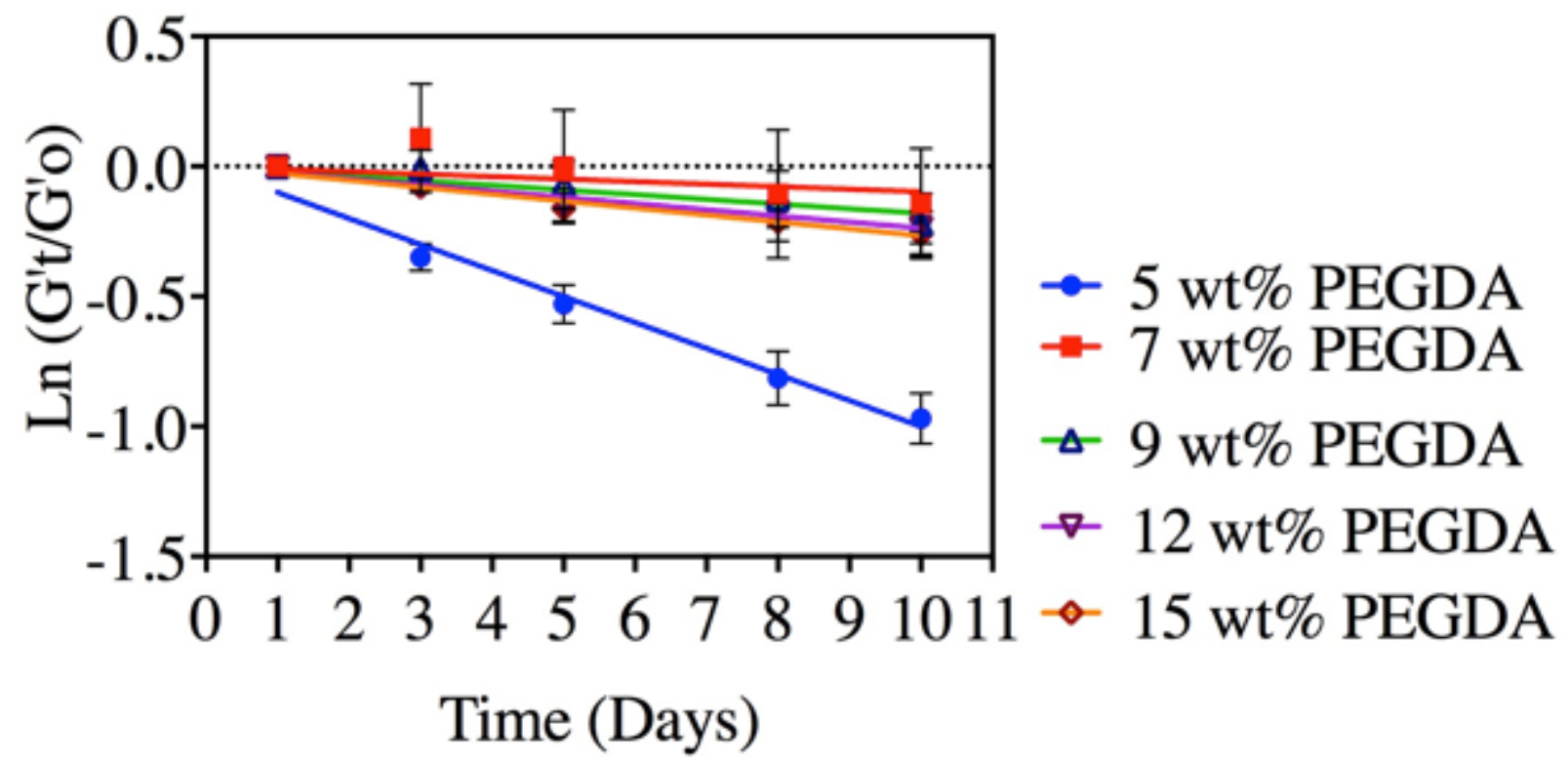

Figure 3: Plot of $\operatorname{Ln}\left(G^{\prime} / G^{\prime} 0\right)$ as a function of time. The linear relation indicated the degradation of thiol-acrylate hydrogels to follow a pseudo first order degradation kinetics.

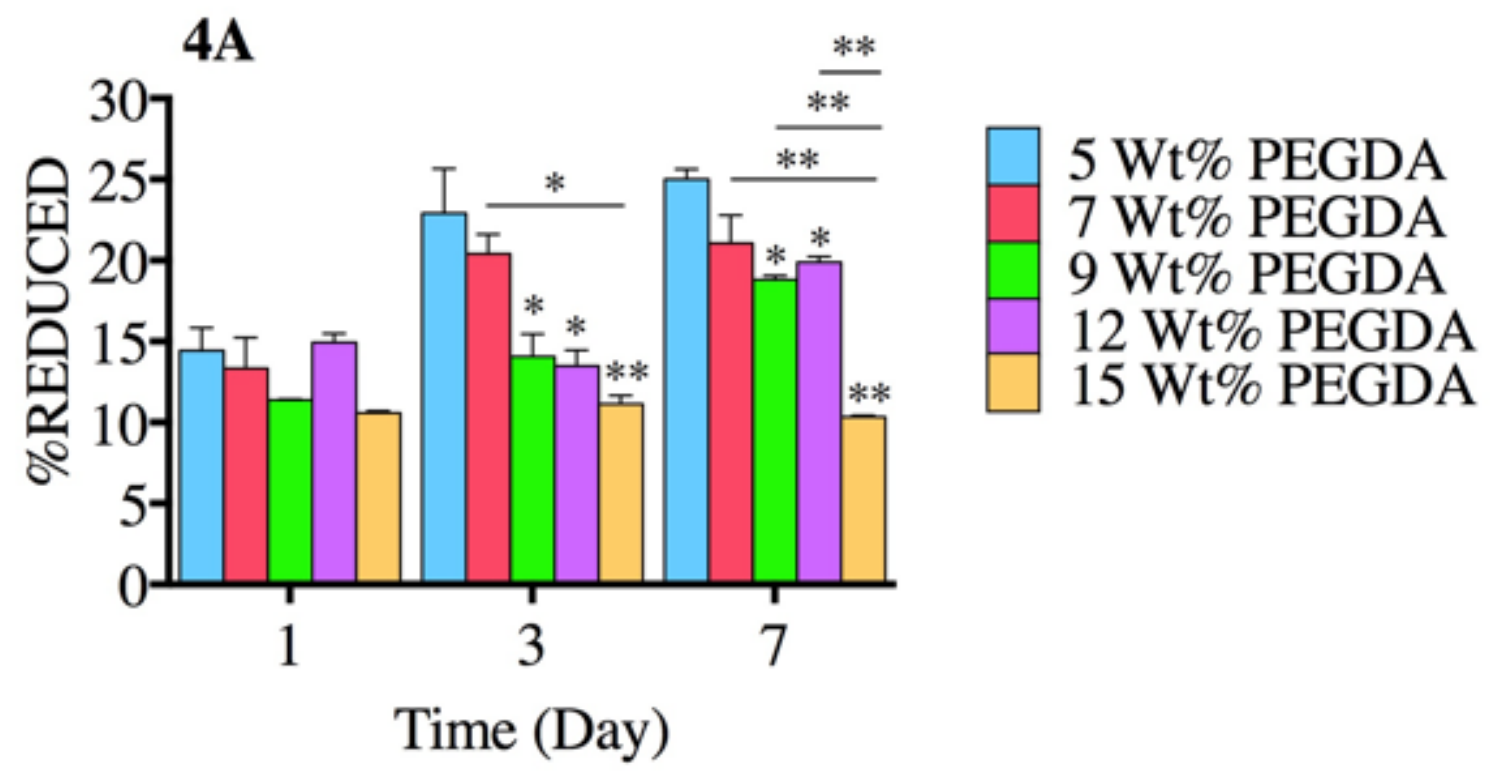




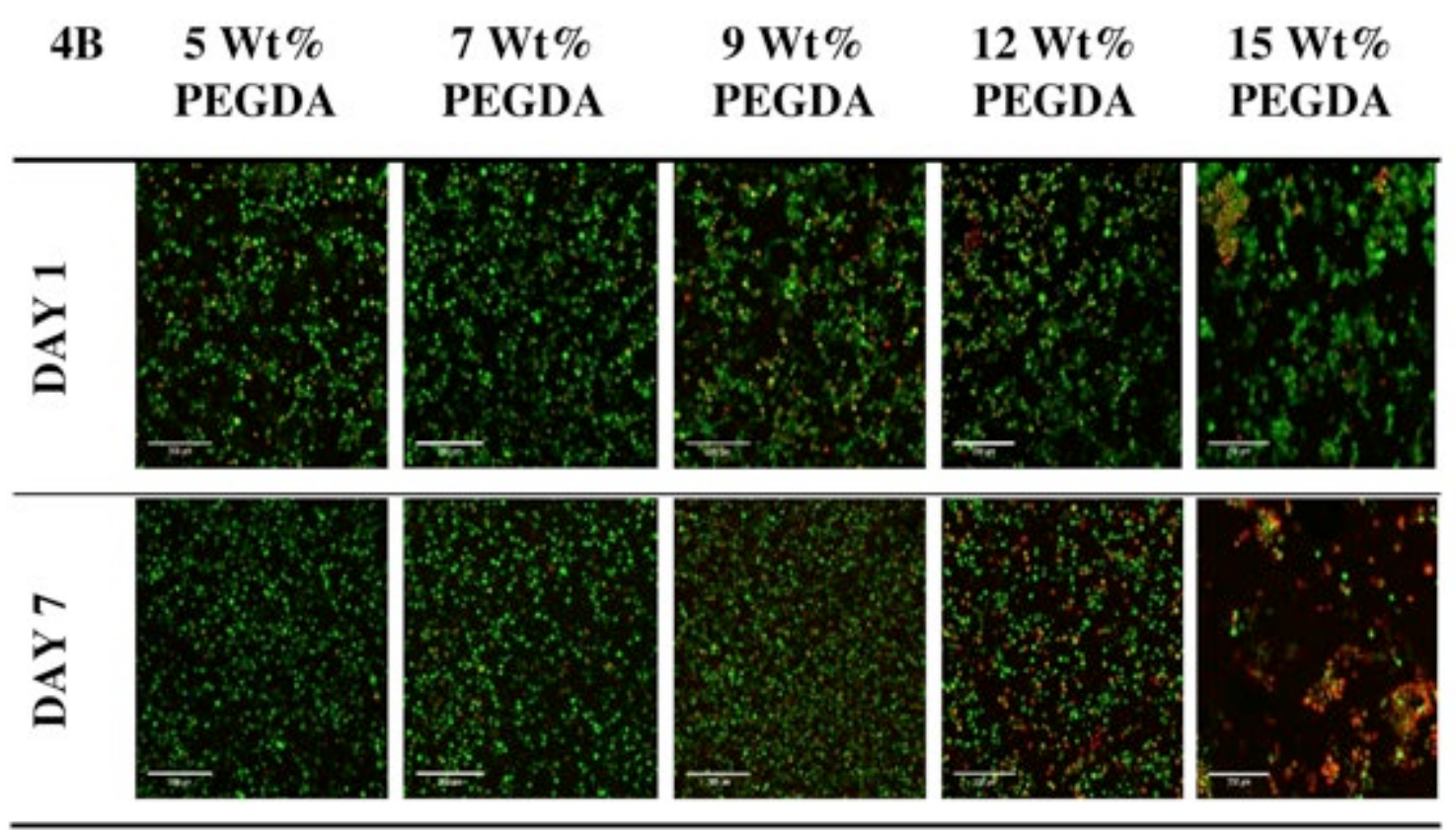

Figure 4: Three-dimensional culture of MC3T3-E1 in visible light cured PEGDA thiolacrylate hydrogels with different PEGDA macromer concentrations. (A) Cell metabolic activity at days 1, 3 and 7 for all PEGDA groups. (B) Confocal microscopy images of encapsulated MC3T3-E1 cells stained with Live/Dead staining at day 1 and day 7. Scale bar is $100 \mu \mathrm{m}\left({ }^{*} \mathrm{p}<0.05,{ }^{* *} \mathrm{p}<0.01\right)$.

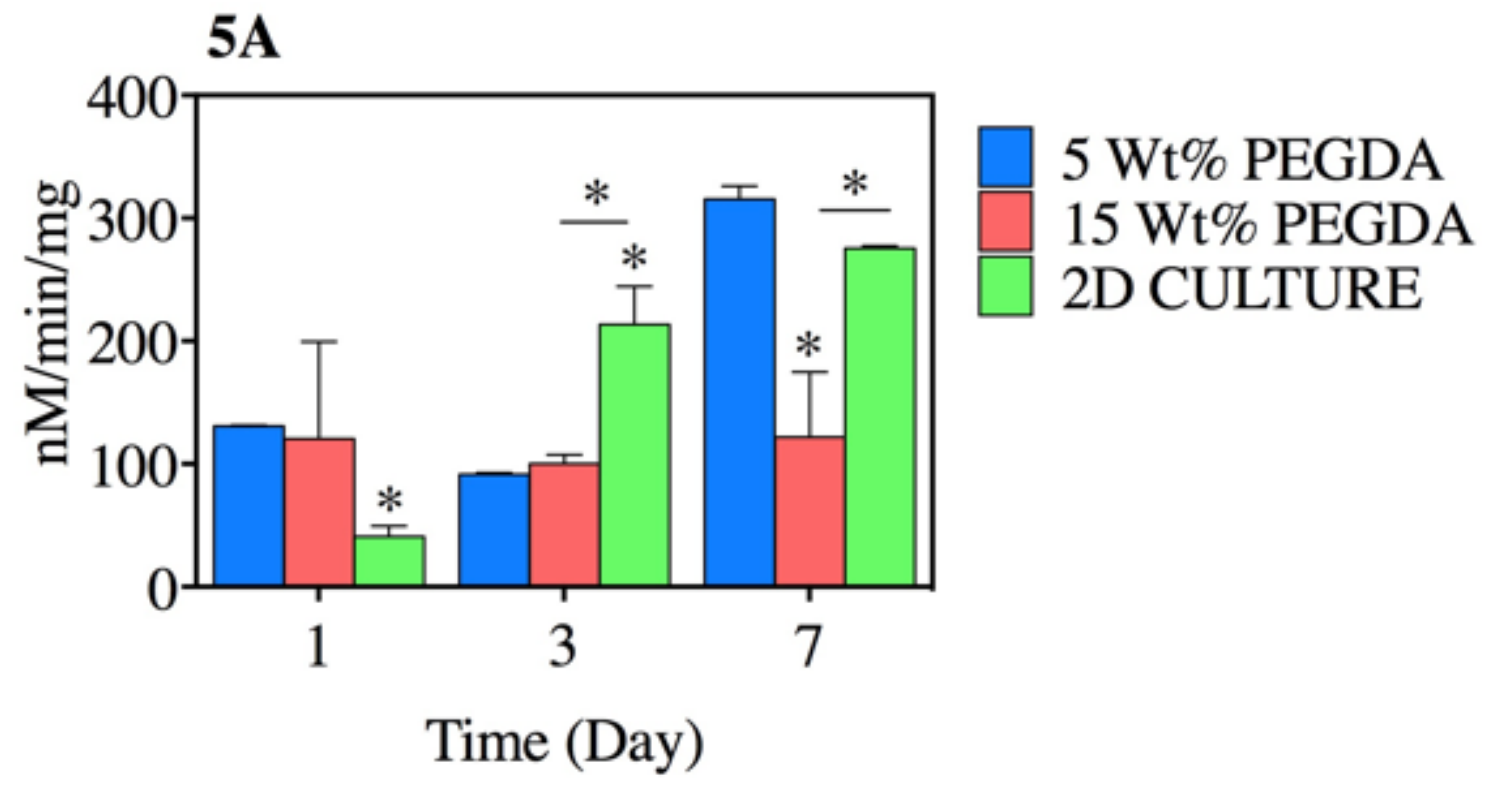




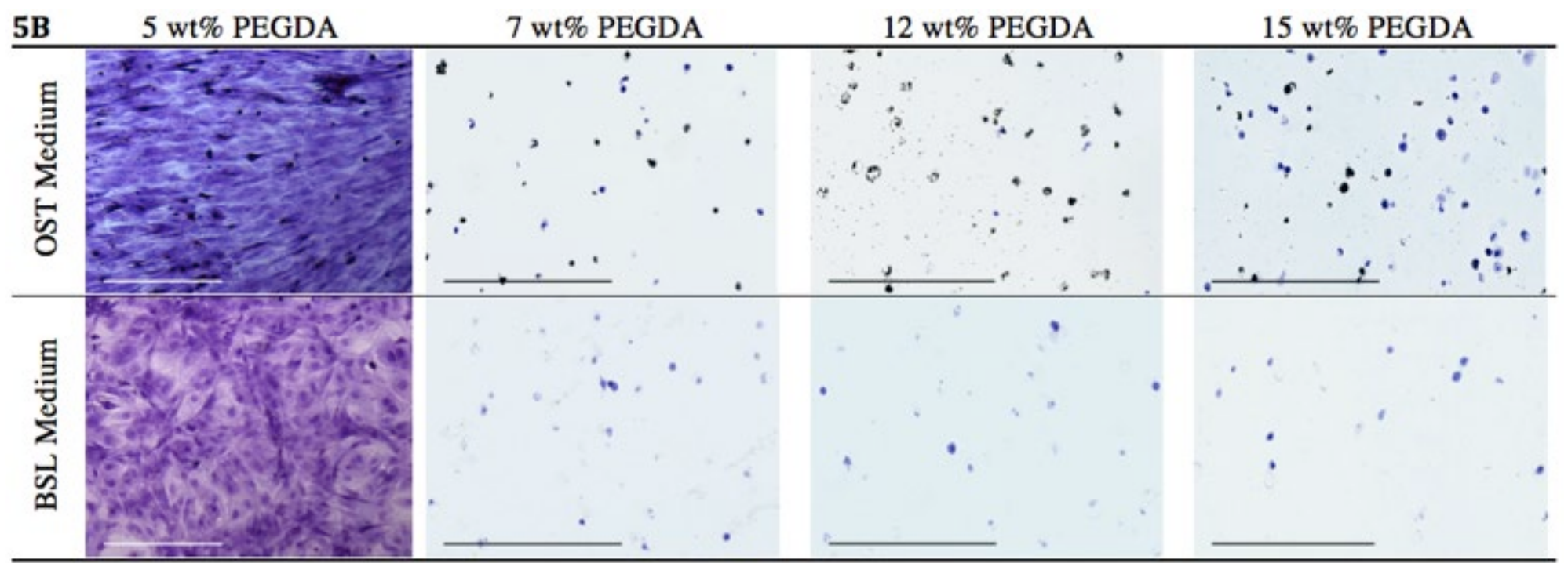

Figure 5: Osteogenic differentiation of MC3T3-E1 encapsulated in PEGDA hydrogels. (A) Normalized ALP activity of encapsulated MC3T3-E1 ( $<$ 0.05). (B) von Kossa, stained calcium in black, and cells in blue. Double staining demonstrates pericellular mineralization of cell cultured in osteogenic medium compared to PEGDA groups cultured in basal medium. Scale bar is $300 \mu \mathrm{m}$.

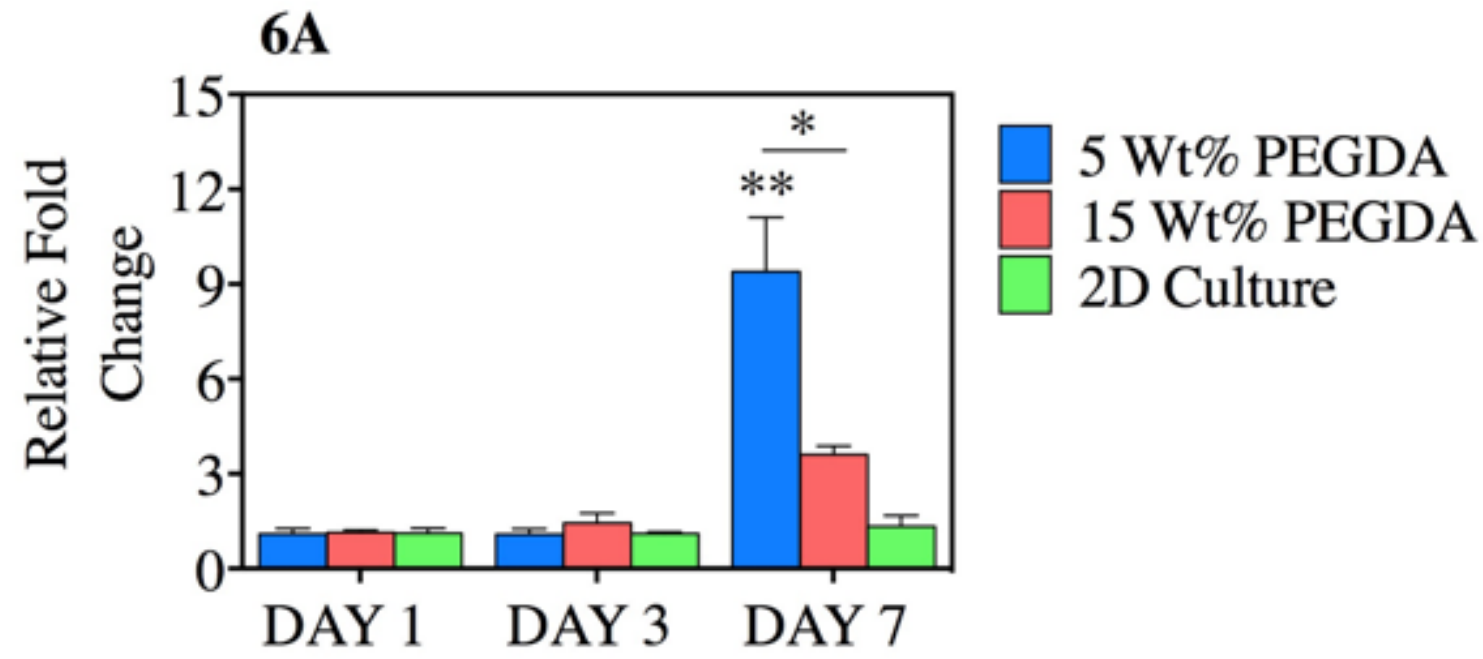




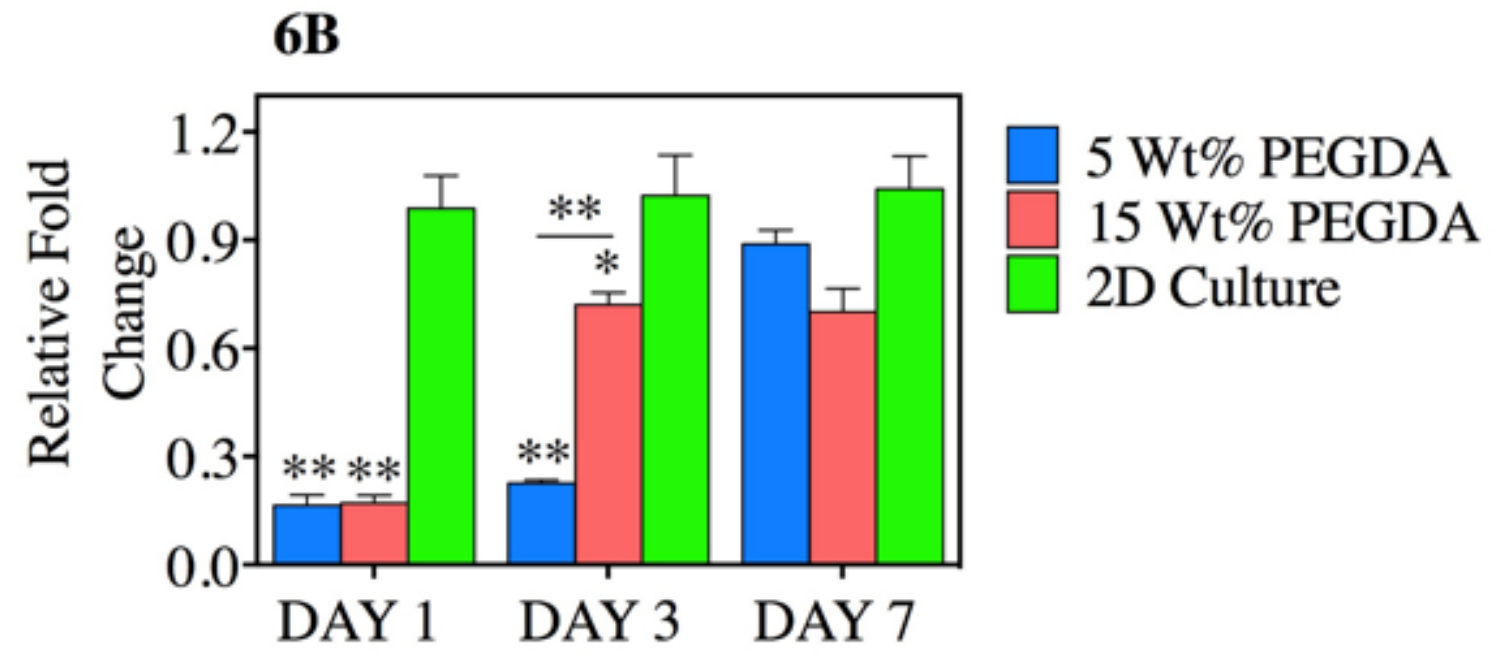

Figure 6: Reverse Transcriptase -qPolymerase Chain Reaction of MC3T3-E1cells encapsulated in PEGDA hydrogels (A) The early osteogenesis marker, RUNX2, was positively upregulated in $5 \mathrm{wt} \%$ PEGDA group and the 2D culture of MC3T3-E1. (B) Type I collagen expression at day 3.(* $\left.\mathrm{p}<0.05,{ }^{* *} \mathrm{p}<0.01\right)$.

\section{References}

ANSETH, K. S., METTERS, A. T., BRYANT, S. J., MARTENS, P. J., ELISSEEFF, J. H. \& BOWMAN, C. N. 2002. In situ forming degradable networks and their application in tissue engineering and drug delivery. J Control Release, 78, 199-209.

BAHNEY, C. S., LUJAN, T. J., HSU, C. W., BOTTLANG, M., WEST, J. L. \& JOHNSTONE, B. 2011. Visible light photoinitiation of mesenchymal stem cell-laden bioresponsive hydrogels. Eur Cell Mater, 22, 43-55; discussion 55.

BRYANT, S. J. \& ANSETH, K. S. 2002. Hydrogel properties influence ECM production by chondrocytes photoencapsulated in poly(ethylene glycol) hydrogels. $J$ Biomed Mater Res, 59, 63-72. 
BRYANT, S. J., NUTTELMAN, C. R. \& ANSETH, K. S. 2000. Cytocompatibility of UV and visible light photoinitiating systems on cultured NIH/3T3 fibroblasts in vitro. J Biomater Sci Polym Ed, 11, 439-57.

BURDICK, J. A., CHUNG, C., JIA, X., RANDOLPH, M. A. \& LANGER, R. 2005. Controlled degradation and mechanical behavior of photopolymerized hyaluronic acid networks. Biomacromolecules, 6, 386-91.

CHAN, B. P. \& LEONG, K. W. 2008. Scaffolding in tissue engineering: general approaches and tissue-specific considerations. Eur Spine J, 17 Suppl 4, 46779.

D'ESTE, M. \& EGLIN, D. 2013. Hydrogels in calcium phosphate moldable and injectable bone substitutes: Sticky excipients or advanced 3-D carriers? Acta Biomater, 9, 5421-30.

ELBERT, D. L. \& HUBBELL, J. A. 2001. Conjugate addition reactions combined with free-radical cross-linking for the design of materials for tissue engineering. Biomacromolecules, 2, 430-41.

FAIRBANKS, B. D., SCHWARTZ, M. P., BOWMAN, C. N. \& ANSETH, K. S. 2009. Photoinitiated polymerization of PEG-diacrylate with lithium phenyl-2,4,6trimethylbenzoylphosphinate: polymerization rate and cytocompatibility. Biomaterials, 30, 6702-7.

FRATZL-ZELMAN, N., FRATZL, P., HORANDNER, H., GRABNER, B., VARGA, F., ELLINGER, A. \& KLAUSHOFER, K. 1998. Matrix mineralization in MC3T3-E1 cell cultures initiated by beta-glycerophosphate pulse. Bone, 23, 511-20.

HAO, Y. \& LIN, C. C. 2014. Degradable thiol-acrylate hydrogels as tunable matrices for three-dimensional hepatic culture. J Biomed Mater Res A, 102, 3813-27.

HAO, Y., SHIH, H., MUNOZ, Z., KEMP, A. \& LIN, C. C. 2014. Visible light cured thiolvinyl hydrogels with tunable degradation for 3D cell culture. Acta Biomater, $10,104-14$.

HERN, D. L. \& HUBBELL, J. A. 1998. Incorporation of adhesion peptides into nonadhesive hydrogels useful for tissue resurfacing. J Biomed Mater Res, 39, 266-76.

LEE, S., TONG, X. \& YANG, F. 2014. The effects of varying poly(ethylene glycol) hydrogel crosslinking density and the crosslinking mechanism on protein accumulation in three-dimensional hydrogels. Acta Biomater, 10, 4167-74.

LI, J. \& KAO, W. J. 2003. Synthesis of polyethylene glycol (PEG) derivatives and PEGylated-peptide biopolymer conjugates. Biomacromolecules, 4, 1055-67.

LIN, C. C. \& ANSETH, K. S. 2009a. Controlling affinity binding with peptidefunctionalized poly(ethylene glycol) hydrogels. Adv Funct Mater, 19, 2325.

LIN, C. C. \& ANSETH, K. S. 2009b. PEG hydrogels for the controlled release of biomolecules in regenerative medicine. Pharm Res, 26, 631-43.

LIN, T. Y., BRAGG, J. C. \& LIN, C. C. 2015. Designing visible light-cured thiol-acrylate hydrogels for studying the HIPPO pathway activation in hepatocellular carcinoma cells. Macromol Biosci.

LIN-GIBSON, S., BENCHERIF, S., COOPER, J. A., WETZEL, S. J., ANTONUCCI, J. M., VOGEL, B. M., HORKAY, F. \& WASHBURN, N. R. 2004. Synthesis and characterization of PEG dimethacrylates and their hydrogels. Biomacromolecules, 5, 1280-7. 
MAZZOCCOLI, J. P., FEKE, D. L., BASKARAN, H. \& PINTAURO, P. N. 2010. Mechanical and cell viability properties of crosslinked low- and high-molecular weight poly(ethylene glycol) diacrylate blends. J Biomed Mater Res A, 93, 558-66.

METTERS, A. \& HUBBELL, J. 2005. Network formation and degradation behavior of hydrogels formed by Michael-type addition reactions. Biomacromolecules, 6 , 290-301.

PANETTA, N. J., GUPTA, D. M., QUARTO, N. \& LONGAKER, M. T. 2009. Mesenchymal cells for skeletal tissue engineering. Panminerva Med, 51, 25-41.

PAPAVASILIOU, G., SONGPRAWAT, P., PEREZ-LUNA, V., HAMMES, E., MORRIS, M., CHIU, Y. C. \& BREY, E. 2008. Three-dimensional pattering of poly (ethylene Glycol) hydrogels through surface-initiated photopolymerization. Tissue Eng Part C Methods, 14, 129-40.

REDDY, S. K., ANSETH, K. S. \& BOWMAN, C. N. 2005. Modeling of network degradation in mixed step-chain growth polymerizations. Polymer, 46, 42124222.

RYDHOLM, A. E., BOWMAN, C. N. \& ANSETH, K. S. 2005. Degradable thiol-acrylate photopolymers: polymerization and degradation behavior of an in situ forming biomaterial. Biomaterials, 26, 4495-506.

RYDHOLM, A. E., REDDY, S. K., ANSETH, K. S. \& BOWMAN, C. N. 2007. Development and characterization of degradable thiol-allyl ether photopolymers. Polymer (Guildf), 48, 4589-4600.

SHIH, H. \& LIN, C. C. 2012. Cross-linking and degradation of step-growth hydrogels formed by thiol-ene photoclick chemistry. Biomacromolecules, 13, 2003-12.

SOKIC, S. \& PAPAVASILIOU, G. 2012. Controlled proteolytic cleavage site presentation in biomimetic PEGDA hydrogels enhances neovascularization in vitro. Tissue Eng Part A, 18, 2477-86.

VERHULSEL, M., VIGNES, M., DESCROIX, S., MALAQUIN, L., VIGNJEVIC, D. M. \& VIOVY, J. L. 2014. A review of microfabrication and hydrogel engineering for micro-organs on chips. Biomaterials, 35, 1816-32.

WANG, H. B., DEMBO, M. \& WANG, Y. L. 2000. Substrate flexibility regulates growth and apoptosis of normal but not transformed cells. Am J Physiol Cell Physiol, 279, C1345-50.

WARD, J. H. \& PEPPAS, N. A. 2001. Preparation of controlled release systems by freeradical UV polymerizations in the presence of a drug. J Control Release, 71, 183-92.

WEBER, L. M., HE, J., BRADLEY, B., HASKINS, K. \& ANSETH, K. S. 2006. PEG-based hydrogels as an in vitro encapsulation platform for testing controlled betacell microenvironments. Acta Biomater, 2, 1-8.

YANG, F., WILLIAMS, C. G., WANG, D. A., LEE, H., MANSON, P. N. \& ELISSEEFF, J. 2005. The effect of incorporating RGD adhesive peptide in polyethylene glycol diacrylate hydrogel on osteogenesis of bone marrow stromal cells. Biomaterials, 26, 5991-8.

YANG, J., WANG, F. \& TAN, T. 2010. Degradation behavior of hydrogel based on crosslinked poly(aspartic acid). Journal of Applied Polymer Science, NA-NA.

ZHANG, H., ZHOU, L. \& ZHANG, W. 2014. Control of scaffold degradation in tissue engineering: a review. Tissue Eng Part B Rev, 20, 492-502. 
ZUSTIAK, S. P. \& LEACH, J. B. 2010. Hydrolytically degradable poly(ethylene glycol) hydrogel scaffolds with tunable degradation and mechanical properties. Biomacromolecules, 11, 1348-57. 